\title{
Finite element simulation of internal flows with heat transfer using a velocity correction approach
}

\author{
B S VARAPRASAD PATNAIK ${ }^{1}$, Y T K GOWDA ${ }^{2}$, \\ M S RAVISANKAR ${ }^{3}$, P A ASWATHA NARAYANA ${ }^{4}$ and \\ K N SEETHARAMU ${ }^{4}$
}

${ }^{1}$ Department of Computational Science, National University of Singapore, Singapore 117543

${ }^{2}$ Department of Mechanical Engineering, PES College of Engineering, Mandya 571 401, India

${ }^{3}$ Department of Mechanical Engineering, Texas A\&M University, College Station, TX 77843, USA

${ }^{4}$ School of Mechanical Engineering, USM, 14300, Nibong Tebal, Malaysia e-mail: \{bsv_patnaik,knseetharamu\}@ @otmail.com

MS received 14 January 2000; revised 9 January 2001

\begin{abstract}
This paper enumerates finite-element based prediction of internal flow problems, with heat transfer. The present numerical simulations employ a velocity correction algorithm, with a Galerkin weighted residual formulation. Two problems each in laminar and turbulent flow regimes are investigated, by solving full Navier-Stokes equations. Flow over a backward-facing step is studied with extensive validations. The robustness of the algorithm is demonstrated by solving a very complex problem viz. a disk and doughnut baffled heat exchanger, which has several obstructions in its flow path. The effect of wall conductivity in turbulent heat transfer is also studied by performing a conjugate analysis. Temporal evolution of flow in a channel due to circular, square and elliptic obstructions is investigated, to simulate the vortex dynamics. Flow past an in-line tube bank of a heat exchanger shell is numerically studied. Resulting heat and fluid flow patterns are analysed. Important design parameters of interest such as the Nusselt number, Strouhal number, skin friction coefficient, pressure drop etc. are obtained. It is successfully demonstrated that the velocity correction approach with a Galerkin weighted residual formulation is able to effectively simulate a wide range of fluid flow features.
\end{abstract}

Keywords. Velocity correction method; finite element simulation; turbulent flows; internal flows; bluff body flows; heat exchangers. 


\section{Introduction}

Most flows of technological interest are turbulent, at least in some regions. For many of these flows, relatively simple prediction methods suffice to produce results of engineering accuracy. However, for high technology applications, more precise tools and procedures are highly essential. Internal flows such as the flow through straight and curved ducts, channels, cascades, diffusers, nozzles, combustion chambers, turbo-machinery stators, rotors etc. are some of the most complex flows encountered in practice.

Fluid dynamics and heat transfer behaviour of laminar and turbulent flows in ducts and channels with obstacles have wide applications in heat exchangers. Design and performance of heat exchangers can be optimized if the quantitative influence of various operating conditions could be pre-determined. Basic understanding of fluid flow and heat transfer forms the primary basis for gaining useful insights. Compared to segmental baffles, disk and doughnut baffles have not enjoyed similar popularity, mainly due to manufacturing problems and the absence of similar information on heat transfer and pressure drop. Hence, conjugate analysis of turbulent flow in a disk and doughnut baffled heat exchanger is chosen as one of the problems for investigation.

Flow past tube banks with a variety of configurations has wide applications, such as heat exchangers, nuclear reactors, boilers, condensers, waste heat recovery systems etc. An understanding of wake behaviour and the associated dynamics for flow about a single cylinder and an array of tubes forms the first step towards better and improved design of heat transfer equipment. Due to smaller flow passages, and a tighter packing of the tube bundle, heat exchanger design range is sometimes well within the laminar flow regime. A common understanding is that turbulent flows provide high heat transfer coefficients and, hence, are desirable for this class of applications. On the contrary, it leads to increased pumping costs, which should be taken into consideration at the design stage. Therefore, laminar flow heat exchangers can also offer substantial weight, volume, space and cost savings. Thus, there is wide interest in the study of fluid friction and heat transfer in heat exchangers where the shell side fluid can be classified as laminar. Apart from heat exchangers (compact and, shell and tube etc.), application of laminar flow theory over tubes has relevance in aerospace, nuclear, biomedical, electronics and instrumentation fields. Such a wide range of practical applications have motivated the analysis on flow past a bundle of cylinders in laminar flow. Further, as a good precursor to this problem, the flow past a single cylinder, as an obstruction in a channel is investigated with extensive comparisons. Flow past a cylinder in a channel is a problem of fundamental importance and has potentially strong application in vortex shedding flow meters. Flow past three basic shapes, viz., square, circular and elliptic configurations, as obstacles in a channel is chosen for the analysis.

With the fast emerging tera-power of the modern day digital computers, numerical simulations have virtually stolen the limelight from the traditional place occupied by experiments for engineering problem-solving, and scientific reliability. Indeed, numerical simulations have emerged as a cheaper alternative in some cases, or at least as complementary in several other situations. Finite element method is one of the most

A list of symbols is given at the end of the paper 
popular tools for engineering analysis and design. It has an enviable generality and a variety of boundary conditions can be implemented with ease. The ability to deal with arbitrary and complex geometries is its major advantage over competing methods. In finite elements, the equations are multiplied by a weight function before they are integrated over the entire domain. This is a distinguishing feature compared to either finite difference or finite volume methods. Finite element method is relatively easy to analyse mathematically, and can be shown to have optimality properties. In the present study, we employ a velocity correction algorithm with a finite element based Galerkin weighted residual formulation. The Reynolds averaged form of the Navier-Stokes equations (RANS) together with a workable turbulence model is the most practical means of computing this class of complex flows in fluid mechanics. Flow past a backward facing step and a disk and doughnut heat exchanger is solved using $k-l$ and $k-\varepsilon$ models. Flow in a channel with a single cylindrical blockage and a bank of cylinders is solved under laminar flow conditions.

\section{Earlier studies}

In a macroscopic sense, laminar flows are well-ordered and are characterized by the sliding of one fluid layer over another. On the contrary, turbulent flows are rather eddying and chaotic even at the macroscopic level. Computational fluid dynamics (CFD) facilitates the numerical simulation of fluid flow features for both laminar and turbulent flows by solving appropriate partial differential equations. In contrast to the revolutionary pace of CFD, developments in turbulence modeling have been only evolutionary and the resulting pace of improvement has frustrated the CFD community (Marvin \& Huang 1998). This frustration largely stems from the lack of clear choice for a general turbulence model. As state-of-the-art computers have advanced, so has the range and size of tasks demanded of them. As larger and much faster machines come along, more complex flow models will be applied. Hence, bigger, faster and less expensive computers will always be sought after. But, if maximum benefit is to be obtained, three major areas need attention. These are computational algorithms, methods of dealing with complex geometries and turbulence models (Ferziger 1987).

Grossly there are three main approaches to turbulent flow simulations: (i) DNS (ii) LES and (iii) Reynolds averaging. It is universally believed that Navier-Stokes (N-S) equations adequately and exactly represent both laminar and turbulent flows. If three-dimensional N$\mathrm{S}$ equations can be solved on a fine enough grid with an exceptionally accurate discretization method, both fine and large scale aspects of turbulence can be captured, which is called direct numerical simulation (DNS) of turbulence. The modeling efforts and simplifications are essentially due to the difficulty in solving the full N-S equations on a very fine grid. In large eddy simulation (LES), only larger eddies can be resolved. Effects of eddies smaller than the mesh size are simulated by sub-grid scale (SGS) models and the predictions are as good as DNS. However, large amount of computational time is still needed to get the statistics. Further, DNS and LES are very expensive and engineers do not want the output of all the three instantaneous velocity components and pressure as a function of $X, Y, Z$ and time, they only need the basic statistics. This brings us to the really sweeping simplification of Reynolds averaging (Bradshaw 1996). Thus, the main task boils down to relating the Reynolds stresses $\left(\overrightarrow{u_{i}^{\prime} u_{j}^{\prime}}\right)$ to the turbulent flow parameters and the mean flow field. 
A variety of turbulent flow models are available in the literature (see Launder \& Spalding 1972; Rodi 1984; Ferziger 1987 and Leschziner 1993). The review by Ferziger (1987) provides a concise but excellent description of various levels of turbulent flow simulations. He has discussed different Reynolds averaged turbulence models, treatment of geometrically complex flows and solution techniques. Correlations, integral methods, one-point and two-point average methods, LES and DNS, represent various levels of turbulent flow simulations. The most popular one-point closure models neglect the effect of molecular viscosity in comparison with the eddy viscosity. Such an assumption is valid only in the fully turbulent regions as it cannot resolve the viscous sub-layer, which is formed close to the solid boundaries. There are two distinct approaches to overcome this drawback. The wall function approach is also called the high Re version, while the other, which incorporates damping functions in the model, is known as the low Re version of turbulence modelling.

Two-equation models are among the most popular turbulence models for scientific and engineering calculations. The celebrated $k-\varepsilon$ model was originally from Jones \& Launder (1972); however, the empirical constants in common use are those recommended by Launder \& Sharma (1974). In these models, two separate transport equations are solved to determine the length of the velocity scales for the eddy viscosity. By doing so, the necessity to specify the length scale on an $a d$ hoc basis can be avoided. Nallasamy (1987) gives an excellent review of turbulent internal flows. The $k-\varepsilon$ model is used in most of the two-dimensional flow calculations reported in the literature. The $k-\varepsilon$ model predicts many flows, including separation and other complex 3-D flow features with an accuracy that is sufficient enough for practical purposes (Rodi 1982). According to Rodi, stress equation models simulate turbulence in a more realistic way and are capable of describing many of the features that defy simulation with an isotropic eddy viscosity model. However, models of this type are rather complex and more expensive. Hence, they are not very suitable for practical applications. Thus, he favours the $k-\varepsilon$ model as the best among the models tested. Taylor et al (1981); Durst \& Tropea (1982) and Isomoto \& Honmd (1989), have studied the problem of turbulent flow over a backward facing step. Formulation and appraisal of prediction methods, and improvements in current design practice for a shell and tube heat exchangers with disk and doughnut baffles, is available in the recent study of Li \& Kottke (1999).

There is a vast amount of literature on bluff body wakes, particularly behind circular and square configurations (see Kovasznay 1949; Ökajima 1982; Blevins 1990; Williamson 1996). Even after over a century of effort, by researchers and engineers, the problem of bluff body flow remains entirely in the descriptive and empirical realm of knowledge. Numerical simulations are not theoretical solutions but are experimental ones, parallel to those realized in the laboratory (Roshko 1993). Systematic experimental and numerical investigations have been undertaken by Ökajima $(1982,1990)$ to study the problem of vortex shedding behind rectangular cylinders of different aspect ratios. Davis et al (1984) have reported both numerical and experimental simulations for flow over a square configuration in a channel. Mukhopadhyay et al (1992) have investigated the influence of a square blockage in a channel by employing a modified version of the MAC method. Braza et al (1986) have distinctly enumerated the chief sources of perturbation, which cause unsteadiness in experimental and numerical simulations. Perry et al (1982) have proposed the concept of alley-way as the mechanism responsible for vortex street 
formation. A visualized account of the shedding patterns is given by Sa \& Chang (1991) and also Patnaik et al (1996). Kelkar \& Patankar (1992) have performed a linear stability analysis and predicted the onset of unsteadiness arising out of vortex shedding. Further, an understanding of the laminar flow past a circular cylinder forms the first step towards understanding the vastly more complicated phenomenon of turbulence (Eaton 1987). Thus, the simulation of flow past a circular cylinder in a channel becomes a mandatory first step to the more complex tube bundle problem. An extensive review on flow past tube banks is available in Zukauskas (1987). Though the advances in computing are on a jet track, the real life practical range of parameters and operating conditions are much more complex than can be numerically simulated. However, these hurdles and complexities have not dampened the spirit of the CFD community. They have made some reasonable assumptions and obtained some useful solutions valid for practical designs. Launder \& Massey (1978) and Fuji et al (1984) have employed a finite difference technique, while Dhaubhadel et al (1986) have used penalty function finite element methods for the simulation of tube bank flows.

There are a variety of fractional step approaches, which can be obtained by appropriately combining pressure, convective and viscous terms in the momentum equations. When primitive variables are used in the governing PDE, there is no direct link between the continuity and momentum equations. To bridge this gap, some mathematical rearrangement of the momentum equations is done and thus the popular Poisson equation for pressure is derived. In the simulation of $\mathrm{N}-\mathrm{S}$ equations, first an intermediate velocity is computed by omitting the pressure gradient, and it is later corrected by including the same. This seminal idea was originally introduced by Chorin (1968) in a finite difference context, which is chiefly reflected in a number of papers (see Donea et al 1982; Gresho et al 1984; Kovacs \& Kawahara 1991; Ramaswamy et al 1992 and Ren \& Utnes 1993). Recently, many other authors have paid further attention to Chorin's original idea and have reported several variations to his method (see Kim \& Moin 1985; Gresho 1990; Natarajan 1992, Zhu \& Sethian 1992). A popular approach is to use an explicit forward Euler time integration and include streamline diffusion (or balancing tensor diffusion) in order to improve stability. A fractional step method is employed in the present study for solving steady/unsteady laminar and turbulent, incompressible Navier-Stokes equations. Galerkin weighted residual formulation is used for spatial discretization to implement the velocity correction algorithm.

\section{Method of solution}

In this section, equations governing the fluid flow domain, procedural steps involved in the velocity correction method, features of the scheme and some numerical issues of relevance are discussed.

\subsection{Governing equations}

The formalism used to derive the governing Reynolds averaged Navier-Stokes equations (RANS) leads to the well-known closure problem (Bradshaw 1981), wherein the fluid motion is described by its mean and a suitably averaged fluctuating motion. The latter is accomplished through turbulence modelling. 
Continuity equation:

$$
\frac{1}{X_{2}}\left[\frac{\partial\left(X_{2} U_{i}\right)}{\partial X_{i}}\right]=0
$$

Momentum equations (in $X_{i}$ direction):

$$
\frac{\partial U_{i}}{\partial \tau}+U_{j} \frac{\partial U_{i}}{\partial X_{j}}=-\frac{\partial P}{\partial X_{i}}+\frac{1}{X_{2}} \frac{\partial}{\partial X_{j}}\left[X_{2}\left(\frac{1+v_{t}}{\operatorname{Re}}\right)\left(\frac{\partial U_{i}}{\partial X_{j}}+\frac{\partial U_{j}}{\partial X_{i}}\right)\right]-\delta_{i, 2} \frac{2 U_{i}}{X_{2}^{2}}\left(\frac{1+v_{t}}{\operatorname{Re}}\right),
$$

where $j=1,2$ for plane and axi-symmetric flows. However, for 2-D plane problems, $X_{2}$ can be chosen as a very large numerical value and the contribution of corresponding radial terms thus become negligible. Therefore, the axisymmetric equations can tend to the twodimensional equations. In the above equations, ' $P$ ' is the static pressure, while ' $v_{t}$ ' is the turbulent momentum diffusivity. The latter (i.e. $v_{t}$ ) is zero for laminar flow problems. Appropriate reference values of velocity $\left(U_{\text {ref }}\right)$ and length $\left(L_{\text {ref }}\right)$ along with kinematic viscosity $(v)$, density $(\rho)$ and turbulent thermal diffusivity $\left(\alpha_{T}\right)$ are used so that the variables are made dimensionless. Time is made dimensionless by using both $L_{\mathrm{ref}}$ and $U_{\text {ref. }}$.

Reynolds number is defined as,

$$
\operatorname{Re}=U_{\text {ref }} L_{\text {ref }} / v .
$$

Energy equation:

$$
\frac{\partial \theta}{\partial \tau}+U_{i} \frac{\partial \theta}{\partial X_{i}}=\frac{1}{X_{2}} \frac{\partial}{\partial X_{j}}\left[X_{2}\left(\frac{1+\alpha_{T}}{\mathrm{Pe}}\right)\left(\frac{\partial \theta}{\partial X_{j}}\right)\right] .
$$

The turbulent thermal diffusivity $\left(\alpha_{T}\right)$, is zero for laminar flow problems, and Peclet number $(\mathrm{Pe})$ is the product of Reynolds and Prandtl numbers. The present simulations are confined to forced convection heat transfer, where the temperature is treated as a passive scalar. The $k-\varepsilon$ model of Lam \& Bremhorst (1981), which is employed in the present study is as follows.

$k$ equation:

$$
\frac{\partial k}{\partial \tau}+U_{i} \frac{\partial k}{\partial X_{i}}=\frac{1}{X_{2}} \frac{\partial}{\partial X_{j}}\left[X_{2} \frac{1}{\operatorname{Re}}\left(1+\frac{v_{t}}{\sigma_{k}}\right)\left(\frac{\partial k}{\partial X_{j}}\right)\right]-Q_{k}=0
$$

where, $Q_{k}$, the source term is given by,

$$
Q_{k}=v_{t} \frac{\partial U_{i}}{\partial X_{j}}\left[\frac{\partial U_{i}}{\partial X_{j}}+\frac{\partial U_{j}}{\partial X_{i}}\right]-\varepsilon
$$

$\varepsilon$ equation: 


$$
\frac{\partial \varepsilon}{\partial \tau}+U_{i} \frac{\partial \varepsilon}{\partial X_{i}}-\frac{1}{X_{2}} \frac{\partial}{\partial X_{j}}\left[X_{2} \frac{1}{\operatorname{Re}}\left(1+\frac{v_{t}}{\sigma_{\varepsilon}}\right)\left(\frac{\partial \varepsilon}{\partial X_{j}}\right)\right]-Q_{\varepsilon}=0
$$

where, the source term $Q_{\varepsilon}$ is given by,

$$
Q_{\varepsilon}=C_{\mu} C_{\varepsilon 1} f_{\mu} f_{1} k \frac{\partial U_{i}}{\partial X_{j}}\left[\frac{\partial U_{i}}{\partial X_{j}}+\frac{\partial U_{j}}{\partial X_{i}}\right]-C_{\varepsilon 2} f_{2} \varepsilon^{2} / k .
$$

In the above equations $k$ is non-dimensionalized with $U_{\mathrm{ref}}^{2}$ and $\varepsilon$ with $U_{\mathrm{ref}}^{3} / L_{\mathrm{ref}}$. Further, constants $C_{\mu}, C_{\varepsilon 1}, C_{\varepsilon 2}, \sigma_{k}$ and $\sigma_{\varepsilon}$ are equal to $0.09,1.44,1.92,1.0$ and 1.3 respectively. The damping functions $f_{\mu}, f_{1}$ and $f_{2}$ are given by:

$$
\begin{aligned}
& f_{\mu}=\left(1-\exp \left(-0.0165 \operatorname{Re}_{k}\right)^{2}\right)\left(1+20.5 / \operatorname{Re}_{T}\right) ; \\
& f_{1}=1+\left(0.05 / f_{\mu}\right)^{3} ; \\
& f_{2}=1-\exp \left(-\operatorname{Re}_{T}^{2}\right) .
\end{aligned}
$$

$\operatorname{Re}_{T}$ and $\mathrm{Re}_{k}$ are defined by the following relations:

$$
\begin{aligned}
& \operatorname{Re}_{T}=\operatorname{Re} k^{2} / \varepsilon ; \\
& \operatorname{Re}_{k}=\operatorname{Re} k^{0.5} y .
\end{aligned}
$$

Turbulent momentum diffusivity is then calculated by,

$$
v_{T}=\operatorname{Re} f_{\mu} C_{\mu} k^{2} / \varepsilon
$$

Unlike $v_{T}$, the turbulent thermal diffusivity $\left(\alpha_{T}\right)$ is not a fluid property. However, it depends on the state of turbulence. In fact, Reynolds analogy between heat and momentum transport also suggests that

$$
\alpha_{T}=v_{T} / \operatorname{Pr}_{T}
$$

In the open literature, there is enough experimental evidence to show that the turbulent Prandtl number $\left(\operatorname{Pr}_{T}\right)$ varies very little across the flow. Even its variation from flow to flow is also small. However, an additional force such as buoyancy could significantly influence this parameter, which is not treated in this study.

\subsection{Velocity correction method}

The velocity correction method was applied, using finite elements in Ravikumaur (1988) by employing a fully explicit time-marching scheme. Ravisankar (1991) and Srinivas (1994) have extensively studied the influence of a variety of turbulent flow models. Patnaik (1998) has employed the modified velocity correction approach of Ren \& Utnes (1993), and studied the flow past a vibrating body (Patnaik et al 1999) and the influence of buoyancy (Patnaik et al 1999). The underlying philosophy behind the scheme can be explained as follows. To start with, pressure terms of the momentum equations are ignored and the fictitious velocities are obtained. Of course, these velocity values will not satisfy 
the continuity equation. Hence, correction is applied after solving the pressure Poisson equation. Thus an iterative scheme is set up. In the present study, time integration is based on Eulers explicit scheme of forward differencing. Spatial discretization is performed by finite element-based Galerkin weighted formulation. The following three steps are repeated with in every iteration.

Step 1: Calculation of pseudo velocities - The intermediate velocities $\left(\widetilde{U}_{i}\right)$ are obtained from (2) by dropping the pressure terms. Thus (2) takes the form,

$$
\frac{\partial \widetilde{U}_{i}}{\partial \tau}=-U_{j} \frac{\partial U_{i}}{\partial X_{j}}+\frac{1}{X_{2}} \frac{\partial}{\partial X_{j}}\left[X_{2}\left(\frac{1+v_{t}}{\operatorname{Re}}\right)\left(\frac{\partial U_{i}}{\partial X_{j}}+\frac{\partial U_{j}}{\partial X_{i}}\right)\right]-\delta_{i, 2} \frac{2 U_{i}}{X_{2}^{2}}\left(\frac{1+v_{t}}{\mathrm{Re}}\right)
$$

Euler's explicit scheme is used to expand the time derivative on the LHS of the above equation as,

$$
\frac{\partial \widetilde{U}_{i}}{\partial \tau}=\frac{\widetilde{U}_{i}-U_{i}^{n}}{\Delta \tau^{n}} .
$$

From (13) and (14) pseudo velocity $\left(\widetilde{U}_{i}\right)$ can be calculated.

Step 2: Pressure Poisson equation - On modifying (2) and (13) and after a mathematical rearrangement, we obtain the following pressure Poisson equation:

$$
\frac{1}{X_{2}}\left[X_{2} \frac{\partial^{2} P^{n}}{\partial X_{i}^{2}}\right]=\frac{1}{X_{2}} \frac{1}{\Delta \tau^{n}}\left(\frac{\partial X_{2} \widetilde{U}_{i}}{\partial X_{i}}\right)
$$

It should be pointed out that, turbulence terms have been ignored in the derivation of the above equation, which is fairly typical.

Step 3: Velocity correction - The velocities for the next time step $U_{i}^{n+1}$ are obtained by correcting the pseudo velocities $\left(\widetilde{U}_{i}\right)$ using the pressure field obtained in step (2):

$$
U_{i}^{n+1}=\widetilde{U}_{i}-\Delta \tau\left(\partial P^{n} / \partial X_{i}\right) .
$$

Heat is treated as a passive scalar. The velocity distribution of the current iteration and the temperature distribution of the previous iteration are employed to obtain the temperature field as follows.

$$
\theta^{n+1}=\theta^{n}+\Delta \tau\left[-U_{i} \frac{\partial \theta}{\partial X_{i}}+\frac{1}{X_{2}} \frac{\partial}{\partial X_{j}}\left[X_{2}\left(\frac{1+\alpha_{T}}{\mathrm{Pe}}\right)\left(\frac{\partial \theta}{\partial X_{j}}\right)\right]\right] .
$$

The Galerkin formulation uses the shape functions as the weight functions. The finite element shape functions are discussed by Fletcher (1984) and Segarlind (1984). Linear triangular elements (with $i, j, k$ as subscripts) are used to represent $U, V, P, \theta, k$ and $\varepsilon$, which are approximated as: 


$$
\begin{aligned}
& U=N_{i} U_{i}+N_{j} U_{j}+N_{k} U_{k} ; \\
& V=N_{i} V_{i}+N_{j} V_{j}+N_{k} V_{k} ; \\
& P=N_{i} P_{i}+N_{j} P_{j}+N_{k} P_{k} \\
& \theta=N_{i} \theta_{i}+N_{j} \theta_{j}+N_{k} \theta_{k} ; \\
& k=N_{i} k_{i}+N_{j} k_{j}+N_{k} k_{k} ; \\
& \varepsilon=N_{i} \varepsilon_{i}+N_{j} \varepsilon_{j}+N_{k} \varepsilon_{k} .
\end{aligned}
$$

Global matrices are formulated separately to solve for $U, V, P, k, \varepsilon$ and $\theta$. The discretized system of equations for the three steps of the velocity correction method can be written as follows.

Step 1:

$$
\mathbf{M} \tilde{\mathbf{U}}=\mathbf{M} \mathbf{U}^{n}-\Delta \tau \mathbf{S} \mathbf{U}^{n} / \operatorname{Re}-\Delta \tau \mathbf{A}^{n} \mathbf{U}^{n}+\Delta \tau \ddot{\mathbf{Y}}_{\mathbf{U}}^{n}
$$

Step 2:

$$
\mathbf{S} \mathbf{P}^{n}=(1 / \Delta \tau) \mathbf{D} \tilde{\mathbf{U}}+\ddot{\mathbf{Y}}_{\mathbf{P}}^{n}
$$

Step 3:

$$
\mathbf{M} \mathbf{U}^{n+1}=\mathbf{M} \widetilde{\mathbf{U}}-\Delta \tau \mathbf{D} \mathbf{P}^{n}
$$

Here, $\mathbf{U}^{n}$ and $\mathbf{U}^{n+1}$ are the global vectors containing all nodal values of velocities at $n$th and $(n+1)$ th time steps respectively. $\mathbf{M}$ is the mass matrix, which is lumped by summing up the rows at elemental level, $\mathbf{S}$ is the diffusion matrix, $\mathbf{D}$ is the divergence matrix $\left(\mathbf{D} \widetilde{\mathbf{U}}=\mathbf{D}_{x} \widetilde{\mathbf{U}}+\mathbf{D}_{y} \tilde{\mathbf{V}}\right)$ and $\mathbf{A}$ is the advection matrix. The elemental matrices associated with (19)-(21) are evaluated as,

$$
\begin{aligned}
& \mathbf{M}^{\mathbf{e}}=\iint_{\Omega^{e}} N N^{T} \mathrm{~d} X \mathrm{~d} Y, \\
& \mathbf{S}^{\mathbf{e}}=\iint_{\Omega^{e}}\left[\left(\frac{\partial N^{T}}{\partial X}\right)\left(\frac{\partial N}{\partial Y}\right)+\left(\frac{\partial N}{\partial Y}\right)\left(\frac{\partial N^{T}}{\partial Y}\right)\right] \mathrm{d} X \mathrm{~d} Y, \\
& \mathbf{D}_{\mathbf{x}}^{\mathbf{e}}=\iint_{\Omega^{e}} N\left(\frac{\partial N^{T}}{\partial X}\right) \mathrm{d} X \mathrm{~d} Y, \quad \mathbf{D}_{\mathbf{y}}^{\mathbf{e}}=\iint_{\Omega^{e}} N\left(\frac{\partial N^{T}}{\partial Y}\right) \mathrm{d} X \mathrm{~d} Y, \\
& \mathbf{A}^{\mathbf{e n}}=\iint_{\Omega^{e}} N\left[U^{n T} N\left(\frac{\partial N^{T}}{\partial X}\right)+V^{n T} N\left(\frac{\partial N^{T}}{\partial Y}\right)\right] \mathrm{d} X \mathrm{~d} Y,
\end{aligned}
$$

where $\left[N^{T}\right]=\left(N_{i}, N_{j}, N_{k}\right)$ is the vector of basis or shape functions for the element. The algebraic system of (19)-(21) together with appropriate boundary conditions can then be 
solved. The shape functions $\left(N_{i}, N_{j}, N_{k}\right)$ for a linear triangular element are defined by the following equation.

$$
\begin{aligned}
& N_{i}=(1 / 2 A)\left(a_{i}+b_{i} X+c_{i} Y\right), \quad i=1,2,3 \\
& a_{i}=X_{j} Y_{k}-X_{k} Y_{j} ; \quad b_{i}=Y_{j}-Y_{k} ; \quad c_{i}=X_{k}-X_{j}, \quad i, j, k=1,2,3 .
\end{aligned}
$$

In the above equations, $X$ and $Y$ refer to the global coordinates of the fluid flow domain. After obtaining velocities, pressures, temperatures, turbulent kinetic energy and its dissipation over the computational domain, the locally available information on primitive variables is used to obtain the required design parameters of interest, such as skin friction coefficient, pressure drop, Strouhal number, Nusselt number etc.

\subsection{Features of the scheme and some numerical issues}

Grid discretization is achieved by a simple technique called iso-parametric mapping, as proposed in Zienkiewicz \& Phillips (1971). In this method, the computational domain is divided into a convenient number of four-sided zones. By employing the curvilinear coordinate system, nodes within and on the boundary of each zone are automatically positioned and referenced to a global co-ordinate system. Each zone is in turn subdivided into triangles with judicious grid spacing. Thus, handling mesh refinement in a specific region becomes easier. Furthermore, a powerful automatic renumbering scheme of Collins (1973) is incorporated to achieve a smaller bandwidth, which in turn, reduces the storage space and computing time. Benim \& Zinser (1985) have reported that linear elements are preferable to higher order elements for turbulent flow problems. This has an added advantage, as it does not require any numerical integration, and consequently saves computational time.

Some advantages of the present scheme are as follows: In steps 1 and 3 (calculation of pseudo-velocities and velocity correction phases), mass lumping is done which makes the stiffness matrix diagonally dominant. The inverse of a diagonal matrix is just the inverse of each element. This approach saves an enormous amount of computational time. The explicit scheme tends to shift the frequency of oscillation upwards, and mass lumping procedure shifts it down (Donea et al 1982). Hence, the combination of these two opposing effects result in a well-matched scheme. The stiffness matrix (formed on the LHS) of the Poisson equation (step 2) does not depend on time and hence, the assembly procedure is done only once in the first iteration. Only the load vector formed on the RHS is updated in each of the iterations. The solution of partial differential equations is sought using finite element method.

An assessment of the accuracy of the numerical simulation procedure is of course of fundamental importance. Hence, it is essential to confirm that the numerical simulation constitutes a desirable representation of the governing PDE's. In a time-marching scheme, time step $(\Delta \tau)$ governs the stability of the algorithm. The time step depends on the mesh size and velocity distribution within the flow domain at that instant of time. In the present scheme, stability of the algorithm is governed by the advection-diffusion term in the velocity prediction phase (step 1 ). Thus, the time step $(\Delta \tau)$ is obtained from, 


$$
\mathrm{C}<\left\langle\frac{\mathrm{Pe}_{c}}{2+\mathrm{Pe}_{c}}\right\rangle,
$$

where, Courant number $(\mathrm{C})=U \Delta \tau / \Delta X$ and the cell Peclet number $\left(\mathrm{Pe}_{c}\right)=U \Delta X / v$. Here, $\Delta \tau$ and $\Delta X$ refer to the minimum allowable time step and smallest spatial interval respectively.

Error in a finite element approximation is defined as the difference between exact and approximate solutions. Actual error can never be calculated, due to non-availability of an analytical solution. However, it is possible to construct an estimate of the error. Such a measurement provides a reliable picture of not only the size of the error, but also the rate of convergence for numerical simulations. A variety of error norms are available for this purpose. In the present study, reduction in incremental error between two successive iterations is taken as the criterion for convergence. The following inequalities can be satisfied for steady state problems:

$$
\begin{aligned}
& U^{n+1}-U^{n} \leq 10^{-9} ; \quad V^{n+1}-V^{n} \leq 10^{-9} ; \\
& P^{n+1}-P^{n} \leq 10^{-9} ; \theta^{n+1}-\theta^{n} \leq 10^{-9} .
\end{aligned}
$$

Steady state solution exists for the backward facing step, disk and doughnut heat exchanger and tube bundle problems under investigation. However, only a steady-periodic solution suffices for flow past a bluff body in a channel. Hence, for these latter class of flows, as the above inequality cannot be satisfied in its totality, the iterative process is stopped after obtaining about 30 vortex shedding cycles. Also, extensive validations are performed with the available analytical and experimental simulations for duct flows (Srinivas et al 1994) and for channels with obstructions (Patnaik 1994). Although the usage of upwinding appears to be popular with some authors, no upwinding is employed in the present finite element simulation. In a general sense, this technique is particularly employed to suppress the numerical oscillations. Upwinding essentially introduces numerical diffusion and thereby effects the accuracy of the method. The authors believe that the method will be devoid of its universal appeal, if upwinding is incorporated in a finite element context. Also, it should be mentioned that, in the context of turbulence modelling, only the standard constants are used, in order to uphold the universality of the constants, even if it means not very good comparison with the experimental data.

In the process of transforming a physical model into a solvable set of algebraic equations, a variety of errors get accumulated. These errors could be due to truncation, round off, approximations, model constants etc. Hence, one has to make sure that the errors are not magnified during the course of a numerical simulation process. There are two systematic ways of error reduction. The first is a grid adapted solution, and the second relies on a series of mesh refinements. The former identifies regions of large gradients for the fluid flow and heat transfer variables, such as velocity, pressure, or temperature, based on error estimates. Though the procedure is elegant and dynamic, it also requires a complex and a reliable algorithm, which could be computationally expensive. However, this has a definite advantage, when the nature of the fluid flow pattern is not known $a$ priori. The second approach is that of choosing a series of mesh systems to arrive at an optimum mesh. The latter procedure is employed in the present simulations. Choosing a judicious mesh system is not very complex as most of the high gradient regions are close to the no-slip boundaries. For example, in a multiply connected domain, like the flow in a 
channel with a cylindrical blockage, three types of mesh densities are employed, to arrive at an optimum mesh. These are as below:
(A) 1464 nodes; 2872 elements.
(B) 2124 nodes; 4298 elements.
(C) 2568 nodes; 5476 elements.

As part of the grid independence study, simulations were conducted, on the above three grid systems. The computational results have shown a maximum difference of $6 \%$ between ' $\mathrm{A}$ ' and ' $\mathrm{B}$ ' type grids, and less than $2 \%$ between ' $\mathrm{B}$ ' and ' $\mathrm{C}$ ' type, with respect to parameters like mean centreline velocities, Nusselt numbers, Strouhal number etc. Further, details are available in Patnaik (1998). Similar studies have been performed for other fluid flow domains to arrive at a sagacious grid system for the particular problem under study.

\section{Results and discussion}

In this section, four problems associated with channel flows of direct relevance and application in heat exchangers are presented. Results of the backward facing step and disk and doughnut baffled heat exchanger are analysed with the aid of turbulence models in high Reynolds number regime. Simulation of channel flows with blockage and flow past a tube bundle in a shell does not include any turbulence model. Due to the highly unsteady and eddying nature of the latter class of flows, simulations are performed in low to moderate Reynolds number regimes, where the resulting fluid flow patterns can be safely assumed to be laminar.

\subsection{Flow over a backward-facing step}

Flow over a backward-facing step is more like sudden expansion in a pipe. However, the former is a plane problem, while the latter is an axisymmetric one. A comprehensive review on the status of numerical modelling for this configuration is given by Nallasamy (1987). Flow separation is a common feature and a zone of recirculation exists on the downstream side of the step wall. The ability of the numerical scheme to correctly predict the length between separation and reattachment points is of crucial importance. However, under-prediction of reattachment length by various turbulence models is a unique feature of this problem. None of the first-order models available in the literature predict the flow in a sudden expansion or flow over a backward-facing step accurately. In order to overcome this under-prediction, many techniques have been adopted in the literature. For example, Autret et al (1987) halve the value of $C_{\mu}$ from the original 0.09 to 0.045 . The smaller value of eddy viscosity essentially means a decreased resistance to the recirculating eddy. This in turn improves the prediction of reattachment length. However, the problem is much more subtle in many ways. The constant $C_{\mu}$ appears in the production term of the $\varepsilon$ equation, and also in the eddy viscosity $\left(v_{t}\right)$, of both $k$ and $\varepsilon$ equations. Taylor et al (1981) employ the value of $C_{\varepsilon 2}=0.18$ in place of the original 1.92. Such a modification essentially leads to an overprediction of $\varepsilon$. It should be pointed out that the constant $C_{\varepsilon 2}$ is associated with the sink term in the dissipation equation. Hence, a gross 
reduction in $C_{\varepsilon 2}$ essentially results in overprediction of $\varepsilon$. This, in turn, leads to underprediction of TKE and the eddy viscosity. Even here, due to coupling between the equations, the net result is not proportional to change in the value of the constant. Therefore, changing constants is not the right approach, as it reduces the universality of the model.

Geometry and boundary conditions: The configuration of the fluid flow domain is shown in figure 1. Entrance length on the upstream side of the step height is chosen as $4 \mathrm{H}$. The length of the channel on the downstream from the expansion is chosen at 20 step heights. The expansion ratio of the channel height on the forward and rearward sides is $2: 3$. The variables are made dimensionless using appropriate combinations of $U_{\mathrm{av}}, H, \mathrm{v}$ and $\alpha$. Hence, Reynolds number of the flow is given by $\operatorname{Re}=U_{\mathrm{av}} H / \mathrm{v}$. Inlet conditions are specified using experimentally measured values of Denham et al (1975). Fully-developed axial velocity and turbulent kinetic energy profiles are specified at the inlet. No-slip condition at the walls and fully developed condition at the exit are imposed. Calculation of $\varepsilon$ at inlet is obtained using the following relation:

$$
\varepsilon_{i n}=\left(C_{\mu}^{9 / 4} k^{3 / 2} / \chi y\right) .
$$

New approach for mixing length specification: The $k-l$ model is a stable model and exhibits monotonic convergence. To obtain good prediction, better mixing length distribution has to be given. A mixing length distribution using the $k-l$ model is available in Taylor et al (1981) for flow over a backward-facing step. This procedure is employed in the present study. However, this distribution does not have a sound physical basis. Hence, a new approach to mixing length specification is suggested and is incorporated in the $k-l$ model. The new specification is purely based on physical arguments. However, no consideration is given to tuning the constants in order to retain the universality of the model. The configuration in figure 1 also depicts a recirculating eddy (dashed). The line $\mathrm{AB}$ demarcates the region into two zones. In the region between the line $\mathrm{AB}$ and the walls, flow takes place in the rearward direction. On the other side, it takes place along the main flow. Approximately, along the line, the velocity of flow changes its direction. Hence, as a

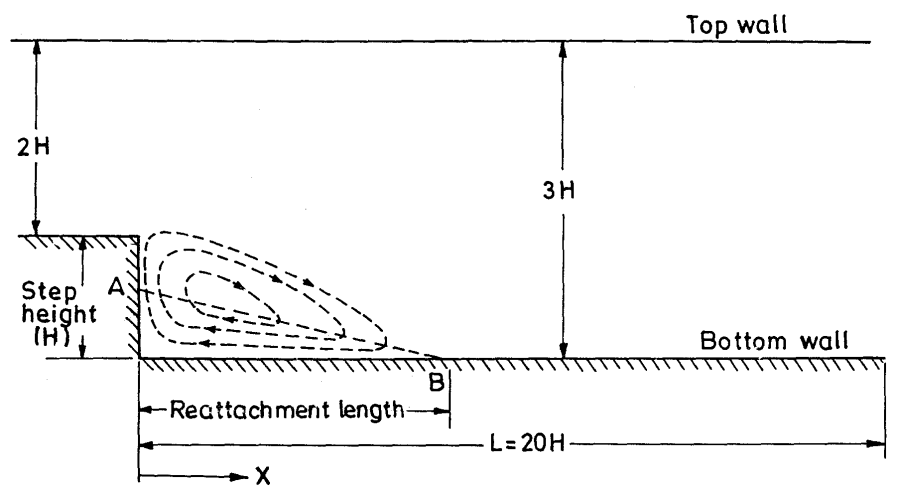

Figure 1. Configuration of the backward facing step. 
first approximation, line $\mathrm{AB}$ can be considered as a line of zero velocity. A more accurate treatment would be to take the immobile centre of the recirculating eddy as a source of reference. Here, in the new approach line, $A B$ is chosen as the centre of reference for mixing length (ML) specification.

$$
M L=\chi(\min (y, \text { normal disance from line } \overline{\mathrm{A}} \overline{\mathrm{B}})) .
$$

Figure 2 depicts the axial velocity profiles at different locations against the experimental result of Denham et al (1975). The three models used in the simulation include $k-\varepsilon, k-l$ with the mixing length specification of Taylor et al (1981), and $k-l$ with the new approach. The predictions agree well with the experimental results. The overall agreement confirms the validity of this new idea. Turbulent kinetic energy profiles are compared in figure 3. The agreement of the $k-\varepsilon$ model with the experimental result is poor. In this figure, TKE values are multiplied by a factor of ten for clarity in presentation. Figure 3 also depicts the superiority of the new mixing length distribution suggested to that by
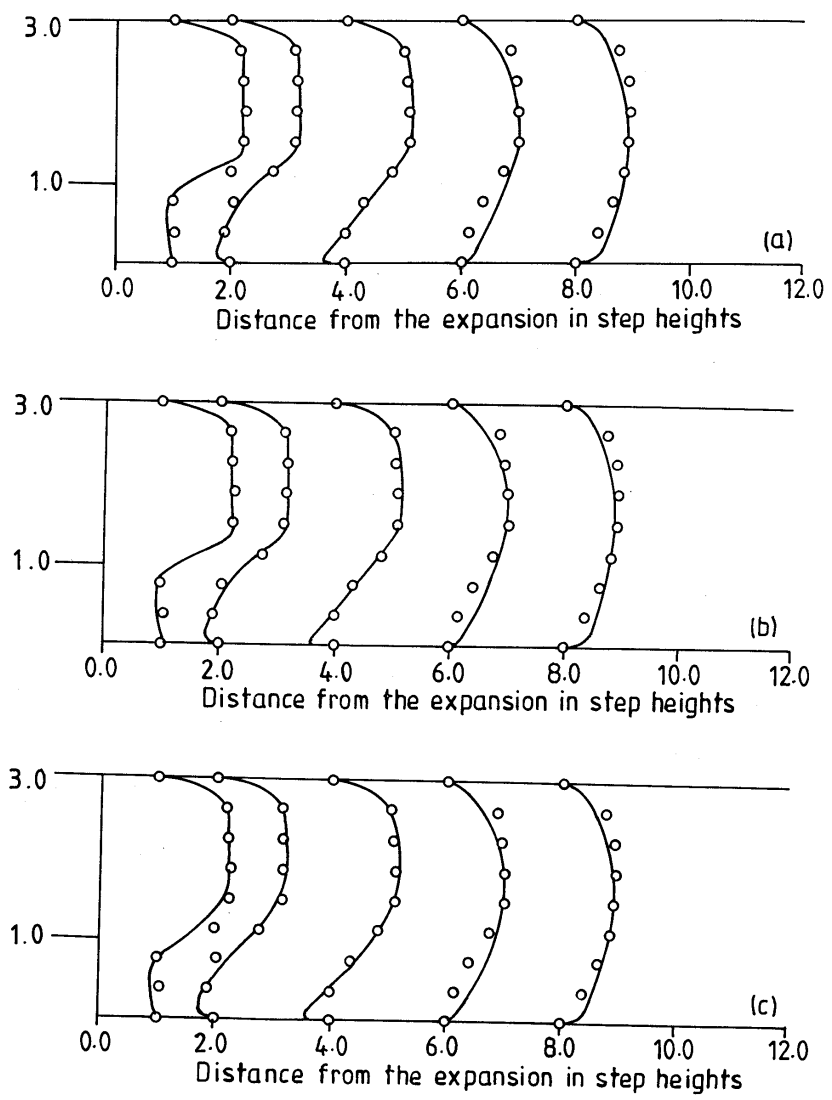

Figure 2. Comparison of axial velocity profiles for $\mathrm{Re}=3025$. (O-O Experimental - Denham et al (1975); - Present study) (a) Using $k-\varepsilon$ model; (b) Using $k-l$ model, mixing length is based on Taylor et al (1981); (c) Using $k-1$ model, with a new approach to mixing length specification. 

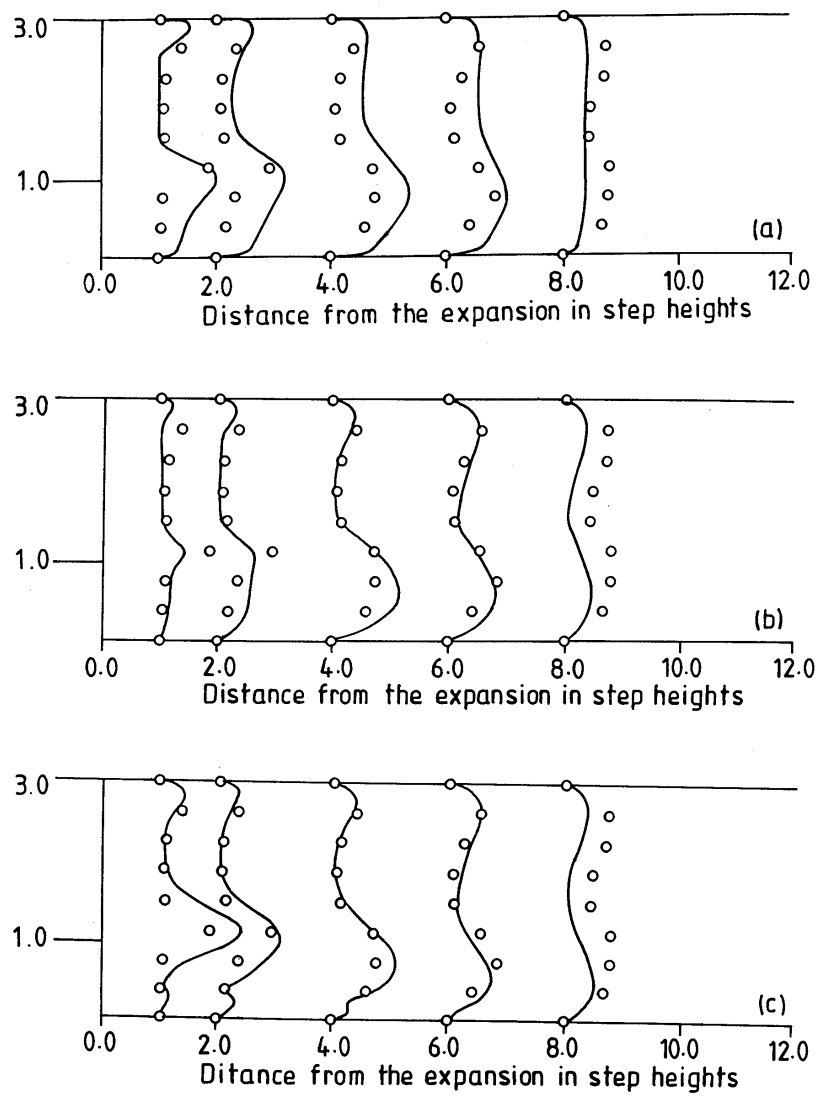

Figure 3. Comparison of turbulent kinetic energy profiles for $\operatorname{Re}=3025$. ( $\mathrm{O}-\mathrm{O}$ Experimental Denham et al (1975); - Present study) (a) Using $k-\varepsilon$ model; (b) Using $k-l$ model, mixing length based on Taylor et al (1981); (c) Using $k-l$ model, with a new approach to mixing length specification.

Taylor et al (1981). Note, particularly, the better agreement near the wall. A comparison of reattachment lengths is available in table 1.

The results agree well with the experimental values and the present predictions are better than those of Taylor et al (1981). However, some amount of underprediction for the reattachment length is a common problem faced by all researchers (Nallasamy 1987). Figure 4 depicts the variation of skin friction coefficient along the channel. Nusselt

Table 1. Comparison of the predicted reattachment length in terms of step height.

\begin{tabular}{lcc}
\hline Model used & Present study & Taylor et al $(1981)$ \\
\hline Standard $k-\varepsilon$ model & $5.60(-6.7 \%$ error $)$ & $4.50(-25.0 \%)$ \\
$k-l$ mixing length from Taylor et al $(1981)$ & $5.90(-1.7 \%$ error $)$ & $5.60(-6.7 \%)$ \\
$k-l$ with new mixing length specification & $5.80(-3.3 \%$ error $)$ & - \\
\hline
\end{tabular}




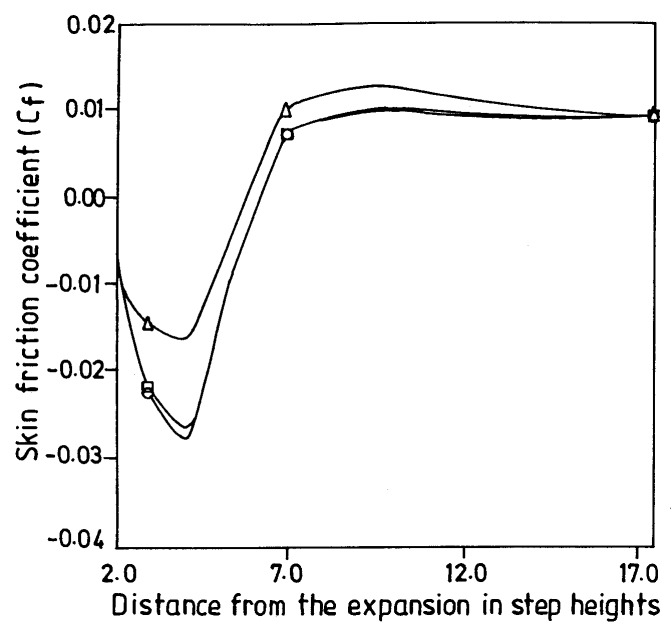

Figure 4. Variation of skin friction coefficient for $\mathrm{Re}=3025 .(\Delta-\Delta k-\varepsilon$ model; O-O $k-l$ model with a new mixing length criterion; $\square-\square k-l$ model of Taylor et al (1981)).

number variation along the channel length, where constant wall temperature is specified, is presented in figure 5. Across the step height, there is a steep drop in the value of skin friction coefficient, while heat transfer coefficient is enhanced, owing to mixing in the recirculating eddy. The predictions from the two $k-l$ approaches have distinct values against the $k-\varepsilon$ model results.

Conclusions: In this section, turbulent flow simulation of flow over a backward-facing step is presented with the aid of three models. The models are: $k-\varepsilon, k-l$ with mixing length specification of Taylor et al (1981), and $k-l$ with a new approach. A new approach to mixing length specification is proposed, purely based on physical reasoning. Axial velocity and turbulent kinetic energy profiles are compared against the available experimental results. Variation of skin friction coefficient and Nusselt number are presented as a function of channel walls. Prediction of reattachment length by the three

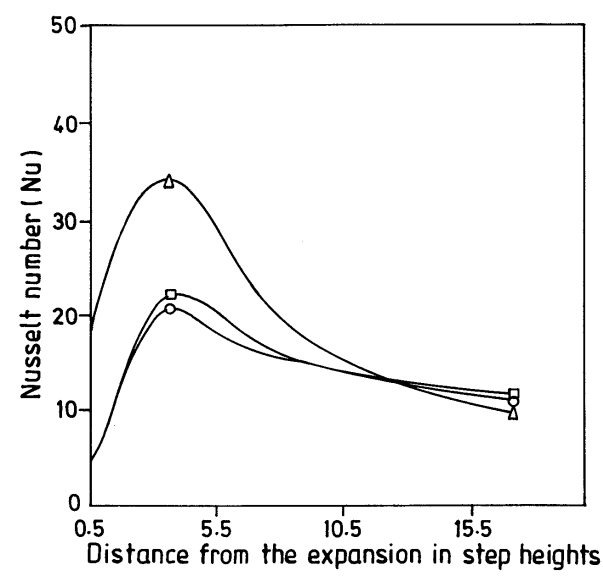

Figure 5. Variation of Nusselt number along the channel walls for $\operatorname{Re}=3025$. $(\Delta-\Delta k-\varepsilon$ model; O-O $k-1$ model with a new mixing length criterion; $\square-\square$ $k-1$ model of Taylor et al (1981)). 


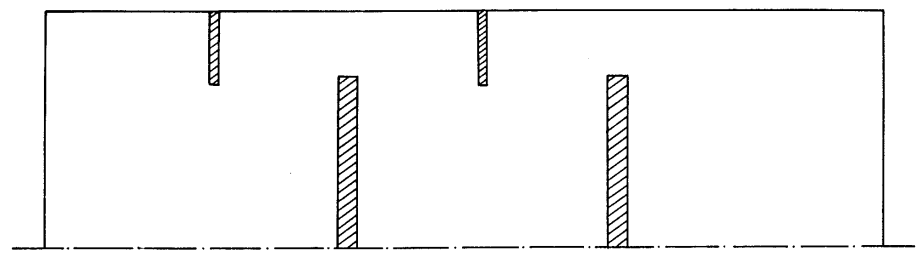

Figure 6. Flow domain of interest for a disk and doughnut baffled heat exchanger.

models is compared. Thus, simulation of flow over a backward-facing step also serves the purpose of extensive validation for the velocity correction algorithm.

\subsection{Disk and doughnut baffled heat exchanger}

In this section, flow in a heat exchanger with disk and doughnut type of baffles is analysed. The shell side of the flow alone is taken into account. Zhang \& Sousa (1990) have studied the momentum transfer aspects for this problem using a finite difference technique. Instead of modelling the walls directly, they use resistivity concept of hydrology to simulate the walls. In their study, the baffles are modelled as infinite hydraulic resistances. However, this approach does not provide any special benefit and is an unnecessary complication. We believe that a conjugate analysis, where the solid wall is also a part of the solution domain, gives a complete picture. Therefore, it is worthwhile to investigate its role under the influence of turbulence and heat transfer.

Conjugate analysis is carried out on a typical disk and doughnut heat exchanger (figure 6) which has a wall thickness of 0.1D. The disks in the flow configuration are not directly involved in any heat transfer; also temperature distribution in the disks is of no relevance as they are completely submerged in the fluid. Axial symmetry (figure 6) is exploited while choosing the problem domain. Inlet conditions are specified as per Zhang \& Sousa (1990). A uniform velocity profile is selected for the inlet. Inlet TKE is taken as 0.012 and dissipation of TKE is given by the following equation,

$$
\varepsilon_{\text {in }}=\left(C_{\mu} k^{3 / 2} / 0.015 D\right) \text {. }
$$

Figure 7 depicts streamline patterns for $\mathrm{Re}=100,000$. Mixing of the flow due to the presence of baffles is clearly brought out in the numerical visual. As can be expected, the presence of a small recirculatory eddy behind the baffles can be seen. Figure 8 shows the skin friction coefficient $\left(C_{f}\right)$ variation for two Reynolds numbers, viz. 45,000 and 100,000.

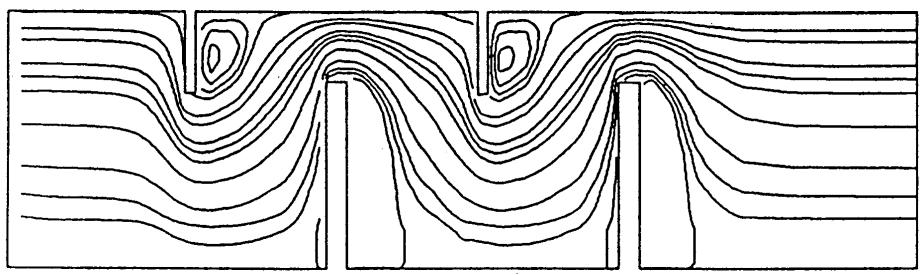

Figure 7. Streamline plot for $\mathrm{Re}=100,000$. 


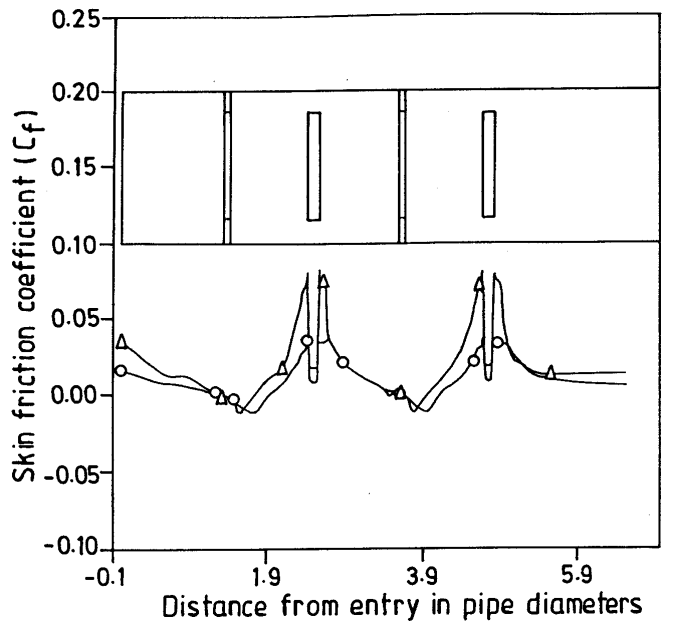

Figure 8. Variation of skin friction coefficient. $\quad\left(\Delta-\Delta \quad \operatorname{Re}=45000 ; \quad O_{-} \mathrm{O}\right.$ $\operatorname{Re}=100,000)$.

This is defined as the ratio of wall shear stress to the kinetic energy of the fluid, which suddenly drops in the location above the disks. Since the flow accelerates in the narrow gap between the wall and the disk, average velocity in that section is very high. Hence, there is a drop in the value of skin friction coefficient, due to a high value on the denominator. Static wall pressure variation along the channel length is shown in figure 9 . Note, particularly, the tremendous increase in pressure drop, which is due to the presence of the disks. Figure 10 depicts the Nusselt number $(\mathrm{Nu})$ variation for $\mathrm{Re}=100,000$. Increase in heat transfer due to the presence of baffles in the flow direction is clearly seen in this figure, along with $\mathrm{Nu}$ variation of a smooth circular pipe (under identical conditions).

In conjugate analysis, wall thickness is part of the solution domain. Analysis is carried out for different values of thermal diffusivity ratio $\left(\alpha_{\text {rat }}\right)$, which ranges from 10 to 100,000 . Figure 11 depicts $\mathrm{Nu}$ variation for different values of $\alpha_{\text {rat }}$ at $\operatorname{Re}=100,000$ and $\operatorname{Pr}=0.7$.

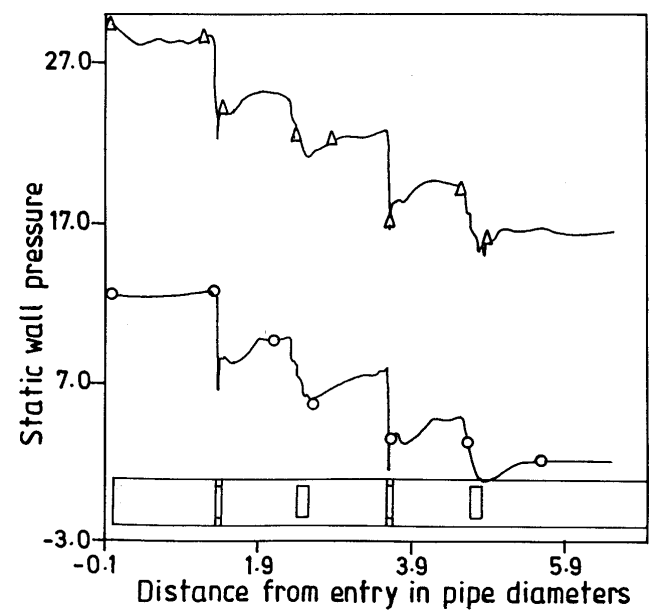

Figure 9. Variation of static wall pressure. $(\Delta-\Delta \operatorname{Re}=45000 ;$ ○-O $\operatorname{Re}=100,000)$. 




Figure 10. Nusselt number variation for $\operatorname{Re}=100,000$ and $\operatorname{Pr}=0.7 . \quad(\Delta-\Delta$ flow in a circular pipe; $\square-\square$ flow in a disk and doughnut heat exchanger).

Nusselt number variations of non-conjugate and conjugate cases with $\alpha_{\text {rat }}=100,000$ are identical. Even for $\alpha_{\text {rat }}=10$ there is no significant distinction. If only the Nusselt number variation is needed, a non-conjugate analysis itself would suffice. However, important differences occur in the variation of temperature on the inner side of the wall. The variation of wall temperature is shown in figure 12. It is clear that, even for $\alpha_{\text {rat }}=100,000$, near the doughnut, the dimensionless temperature is less than one. For other values of $\alpha_{\text {rat }}$, differences are quite high. Table 2 clearly brings out these features, thus substantiating the need for conjugate analysis. Analysis is complete and realistic only when the wall thickness is taken into consideration.

Conclusions: Simulation of fluid flow and heat transfer in a disk and doughnut baffled heat exchanger demonstrates the robustness of the algorithm. A small region of recirculation is prominently noticed behind the disk and doughnut baffles. There is a drop

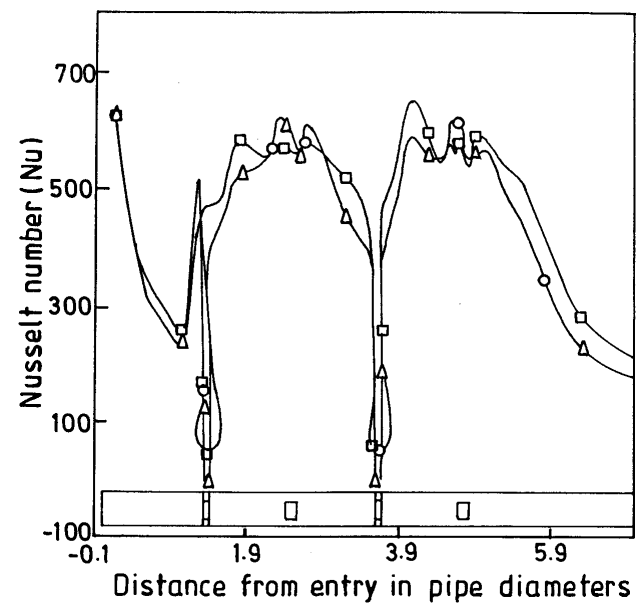

Figure 11. Nusselt number variation with wall temperature for different diffusivity ratios. ( $\square-\square$ $\alpha$ ratio $=10 ; \Delta-\Delta \alpha$ ratio $=100,000 ;$ O-O nonconjugate case). 
Table 2. Exit bulk and wall temperatures, and minimum wall temperature for different values of $\alpha_{\text {rat }}$ at $\operatorname{Re}=100,000$ and $\operatorname{Pr}=0.7$.

\begin{tabular}{lccc}
\hline$\alpha_{\text {rat }}$ & Exit $T_{\text {bulk }}$ & Minimum $T_{W}$ & Exit $T_{W}$ \\
\hline 10 & 0.241 & 0.019 & 0.434 \\
100 & 0.500 & 0.075 & 0.883 \\
1000 & 0.598 & 0.216 & 0.988 \\
10000 & 0.645 & 0.724 & 0.998 \\
100000 & 0.662 & 0.956 & 0.999 \\
$\infty$ & 0.752 & 1.000 & 1.000 \\
\hline
\end{tabular}

in the value of skin friction coefficient and an increase in Nusselt number in the location above the disks. Static wall pressure variation along the length of the channel denotes an increase in pressure drop across the disks. Conjugate analysis is performed by considering wall thickness as part of the solution domain. There is no significant difference in the variation of Nusselt number between conjugate and non-conjugate analysis. However, the exit wall temperature distribution substantiates the need for conjugate analysis.

\subsection{Simulation of vortex shedding in a channel with blockage}

Oscillation of bluff bodies and other structures in transverse flow is essentially due to vortex shedding. An initially smooth and steady flow across a bluff body may bring about damaging oscillations, in cases where the natural frequency of the obstacle is close to the shedding frequency of the vortices. An alternating deflection influences the fluid flow patterns of the wake region behind the cylinder. As a result, the induced forces on the bluff body become periodic and culminate in coupling between the fluid and the structure. This can be detected from the oscillation of the bluff body. If the resulting excitation frequency

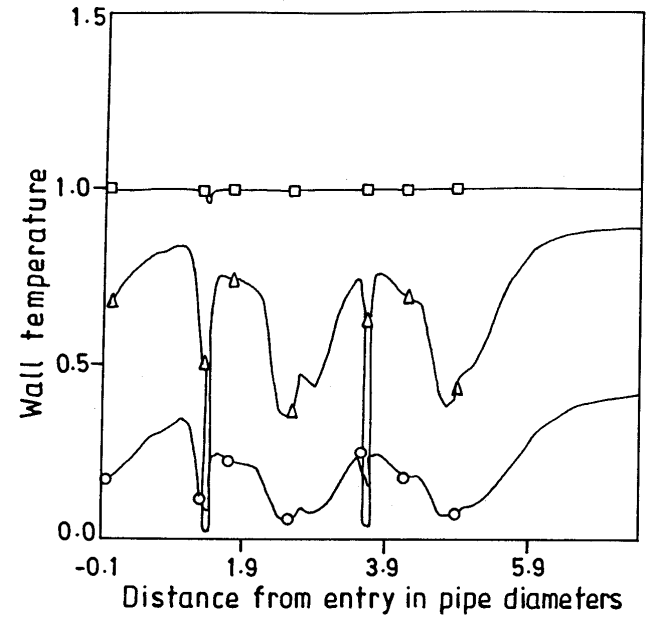

Figure 12. Wall temperature variation for different values of diffusivity ratio at $\mathrm{Re}=$ 100,000 and $\operatorname{Pr}=0.7$. (O-O $\alpha$ ratio $=10 ; \Delta-\Delta$ $\alpha$ ratio $=100 ; \square-\square \alpha$ ratio $=100,000)$. 


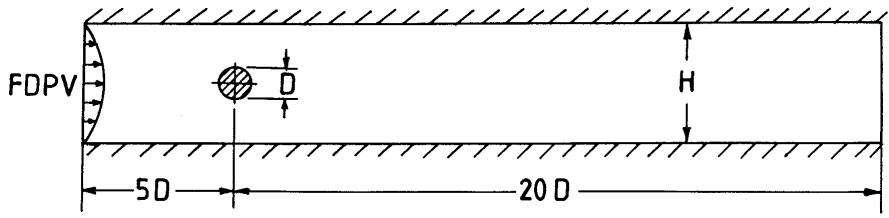

Figure 13. Fluid flow domain of interest.

synchronizes with the natural frequency of the bluff body, the phenomenon of resonance is the obvious outcome. Therefore, the simulation of unsteady flow past bluff bodies has practical relevance. In this section, flow in a channel with circular, square and elliptic blockages is studied. This study has potentially strong and direct application in vortex shedding flowmeters. In this class of applications, the correct value of vortex shedding frequency assumes significance for the measurement of flow rates.

Geometry and boundary conditions: A circular cylinder of diameter $(D)$, acting as a blockage in a channel of height $(H)$, is depicted in figure 13 . The ratio of $D$ to $H$, is blockage ratio. The terms blockage and blockage ratio are interchangeably used in the literature (Perkins \& Leppert 1964). For an unconfined flow, the channel walls are assumed to be at infinity, and hence, blockage is 0.0. For this case, a uniform velocity profile is assumed at the inflow boundary. On the cylinder surface and channel walls, a noslip boundary is enforced. At the exit, a homogenous Neumann boundary condition is applied. A fully developed parabolic velocity profile (FDPV) is imposed at the inlet, when blockage is taken into account. The effect of three blockage ratios, viz., 0.0, 0.1 and 0.25 are studied.

In a channel flow with an obstruction, the wake zone aerodynamics is influenced by three factors, viz., the Reynolds number, blockage ratio and the velocity profile prescribed at the inlet. The primary design objective of such a study would be to find the vortex shedding frequency and hence, the Strouhal number. The downstream side of the cylinder is encompassed with an active zone of fluid dynamical patterns. To study the nature of the fluid flow features along the central axis, time-averaged mean centre line velocities are computed. Figure 14 depicts these values for $\mathrm{Re}=144$ and $\mathrm{BR}=0.0$ in the wake region.

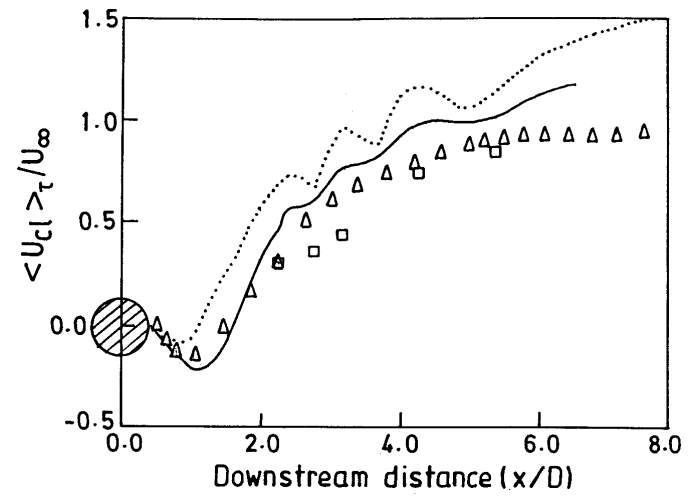

Figure 14. Comparison of mean centreline velocities. ( $\square-\square$ Experimental-Griffin and Votaw (1972); .... Numerical (grid 1) - Chilukuri (1987); — Numerical (grid 2) - Chilukuri (1987); $\Delta-\Delta$ present study). 


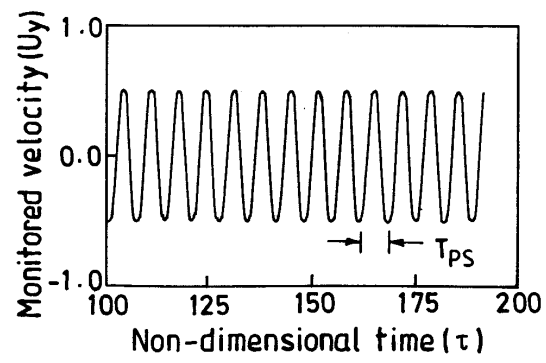

Figure 15. Temporal history of the cross-stream component of monitored velocity.

The negative velocity values depict a zone of reverse flow, immediately behind the cylinder. Experimental observations of Griffin \& Votaw (1972) and Chilukuri (1987) are also presented for the purpose of comparison. The present results compare well with the experimental investigations, especially, in the middle wake region.

The temporal histories for the cross-stream component of velocity $\left(U_{y}\right)$, along the axis of symmetry (in the downstream region), are monitored at several nodal points. A typical plot of the signal traces of the monitored velocity is shown in figure 15 . Time taken from one peak to the other signifies the period of vortex shedding $\left(T_{p s}\right)$ as indicated in the figure. Indeed, $T_{p s}$ can also be obtained from the temporal history of the lift coefficient. Frequency of vortex shedding $\left(f_{v s}\right)$, is obtained as the inverse of time period of vortex shedding $\left(T_{p s}\right)$. Strouhal number, which is a measure of the oscillating fluid flow phenomenon is given by,

$$
S t=\left(f_{v s} D\right) / U_{a v} .
$$

where $f_{v s}$ is the vortex shedding frequency, $D$ is the diameter of the circular cylinder and $U_{a v}$ refers to the average velocity at the inlet. The values of Strouhal number, obtained from the present simulation are compared (figure 16), with the experimental study of Kovasznay (1949) for BR $=0.0$. Numerical results of Chilukuri (1987), Williamson (1988), Karniadakis (1988) and Sa \& Chang (1991) are also presented in the same figure. Good comparison with the earlier investigations can be noticed.

The flow past a circular cylinder is indeed one of the standard benchmark problems, and the simulation is like an acid test for any numerical scheme. Simulating the pattern of flow

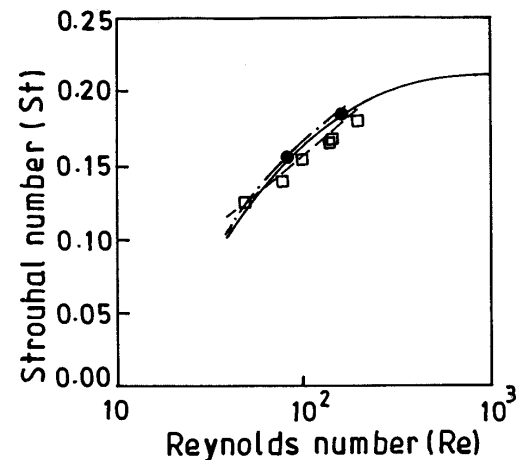

Figure 16. Comparison of Strouhal number against Reynolds number. (- Kovaznay (1949); - - - Williamson (1988); --- Sa and Chang (1991); •-• Chilukuri (1987); $\square-\square$ Present study). 


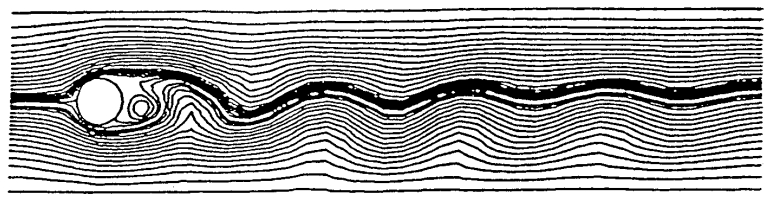

(a)

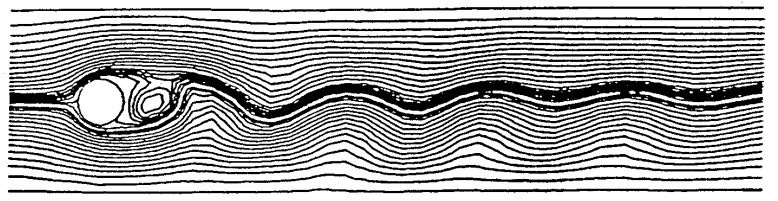

(b)

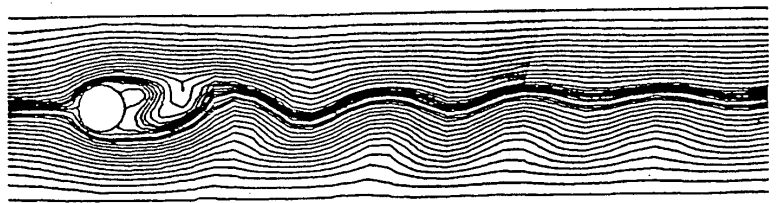

(c)

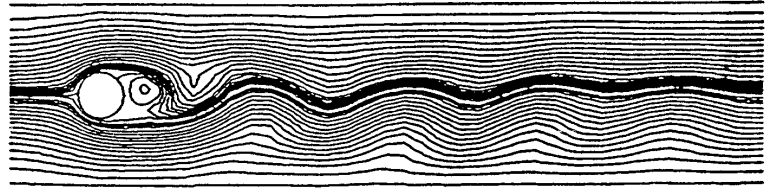

Figure 17. Evolution of streamline patterns over half vortex shedding cycle at $\mathrm{Re}=200 ; \mathrm{BR}=0.25$. (a) Vortex at the bottom; (b) Vortex folded up; (c) Vortex formation at the top (d) Fully grown vortex at the top.

d)

separation, wake region, vortex dynamics etc. renders the problem more fascinating. A typical case of flow field evolution, due to a circular cylindrical blockage, is presented in figure 17 over a half vortex shedding cycle. For a Reynolds number of 200, an eddy is fully formed at the bottom and a sinuous motion can be seen in the downstream (figure 17a). This creates a wake cavity on the topside. Flow entrains through this wake cavity and vortex gets folded up as in figure $17 \mathrm{~b}$. This eddy is subsequently washed away in the wake. With further progress in time, a fully grown eddy is formed on the top, as in figure $17 \mathrm{~d}$. It should be noted that figures $17 \mathrm{a} \& 17 \mathrm{~d}$ visually represent one half of the shedding cycle. Once, two vortices are shed (one each from the bottom and the top of the downstream), one shedding cycle is said to be complete. Again, a new cycle starts and the periodic process repeats. In a physical situation, the destruction in symmetric eddies formed behind the cylinder is inevitable due to multiple perturbation sources, such as nonuniform in-flow conditions, surface roughness of the cylinder, running conditions of the experiment (e.g., small vibrations), turbulence level etc. In a numerical simulation, truncation and round-off errors, and those due to numerical approximation schemes, memory effects etc. are essentially the sources, which play the same role. All these factors eventually lead to the necessary condition of asymmetric disturbance for the generation of vortex shedding (Braza et al 1986). 
Figure 18 depicts the streamlines and isovorticity contours for flow past a square cylinder in a channel with $\mathrm{BR}=0.25$ for $\mathrm{Re}=200$. The undulating nature of the wake can be better understood from the sinuous pattern of the flow lines. Eddy reversal has an interval of half-shedding period, which is a useful measure of vortex shedding frequency. Note particularly the staggered pattern of the vortex street in the isovorticity contours. Figure 19 depicts the streamline patterns for an elliptic cylinder, which has a major to minor axis ratio of 2:1. Here, the Reynolds number is 200 and $B R=0.25$. The serpentine nature of the flow is well established (figure 19). Table 3 helps in understanding variations of Strouhal number among the three basic configurations, viz., circular, square and elliptic cylinders. The square cylinder with its prominent sharp corners has fixed separation points. This makes the flow diverge further and creates a wide wake. Larger wake means higher form drag and smaller value of vortex shedding frequency or Strouhal number. On the other hand, elliptic and circular configurations do not have fixed separation points due to the absence of sharp corners. Their wake region is narrow and eddies can shed faster (for the same velocity). It should be pointed out that the elliptic configuration is more conducive to faster vortex shedding and facilitates it. Hence, square, circular and elliptic configurations are in the decreasing order of their bluffness. For a given configuration, the higher the blockage, the higher is the Strouhal number. These aspects are very well supported from the values obtained in the table.
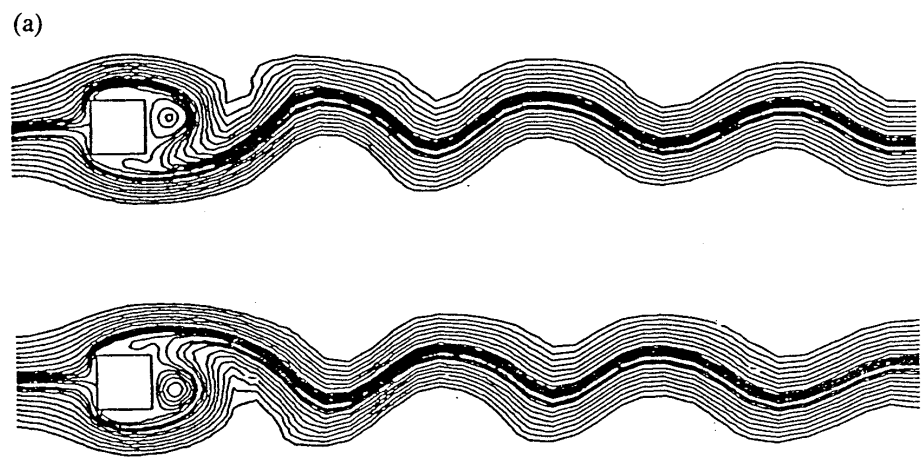

(b)
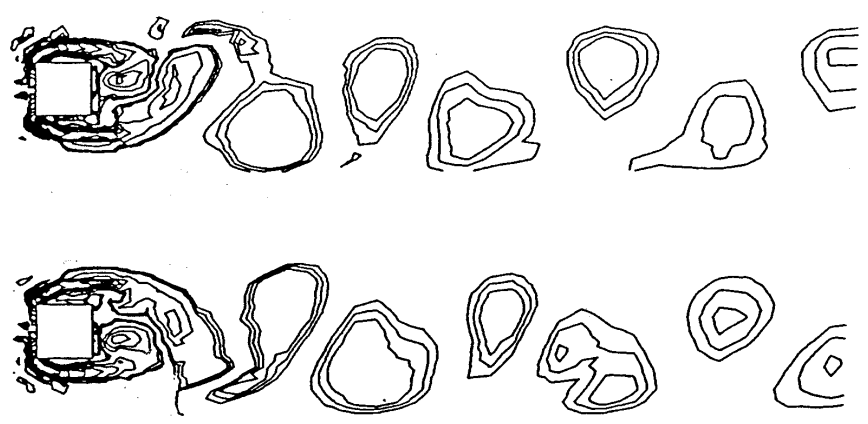

Figure 18. Fluid flow patterns at half vortex shedding cycle apart for a square cylinder $(\operatorname{Re}=200$ and BR = 0.25); (a) Streamlines; (b) Isovorticity contours. 


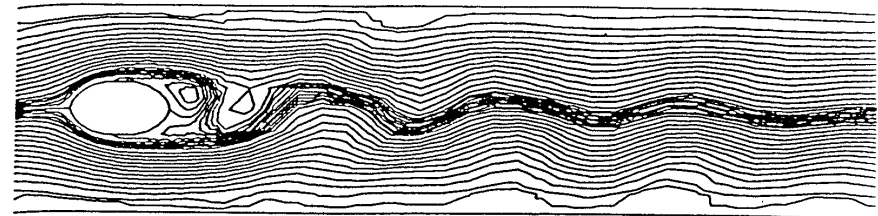

(a)

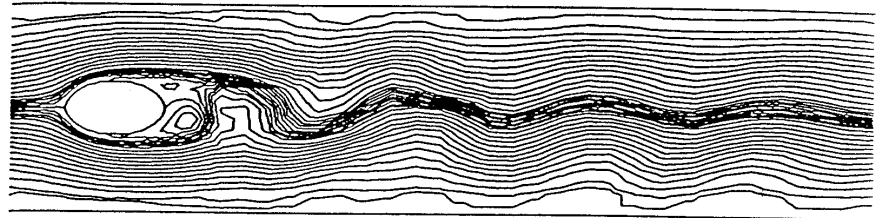

(b)

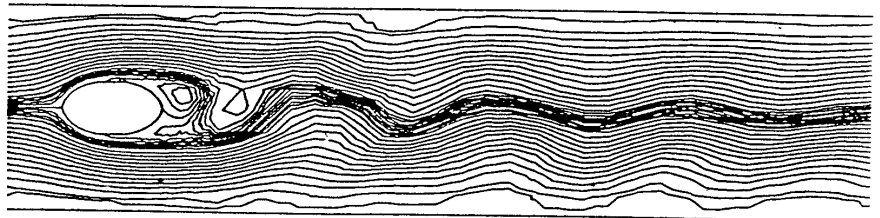

(c)

Figure 19. Streamlines for flow past an elliptic cylinder $(\operatorname{Re}=200$ and $B R=0.25)$.

Having successfully simulated the vortex shedding behind bluff bodies, it should be pointed out that, for $\operatorname{Re}>180$, Williamson (1988) has shown a transition to three dimensionality. Hence, fluid flow features remain two-dimensional only below a Reynolds number, that is close to this value.

Conclusions: Flow past an obstruction in a channel is a good precursor to the flow past a bundle of cylinders. Finite element simulations are performed for three cylinder configurations under blockage ratios of $0.0,0.10$ and 0.25 . Numerical flow visuals depict the oscillating nature of the Kármán vortex street in the downstream. The Strouhal periodicities which are obtained, provide a measure of the oscillating nature of the wake. Square, circular and elliptic configurations are found to be in the decreasing order of their bluffness. Thus, the velocity correction procedure could simulate the complex nature of the vortex dynamics, which is indeed a severe test for the algorithm.

Table 3. Variation of Strouhal number with blockage ratio for $\operatorname{Re}=200$.

\begin{tabular}{lccc}
\hline $\begin{array}{l}\text { Blockage } \\
\text { ratio }\end{array}$ & $\begin{array}{c}\text { Square } \\
\text { cylinder }\end{array}$ & $\begin{array}{l}\text { Circular } \\
\text { cylinder }\end{array}$ & $\begin{array}{c}\text { Elliptic } \\
\text { cylinder }\end{array}$ \\
\hline 0.0 & 0.154 & 0.192 & 0.228 \\
0.1 & 0.168 & 0.213 & 0.252 \\
0.25 & 0.183 & 0.255 & 0.273 \\
\hline
\end{tabular}




\subsection{Flow past a tube bank in a channel}

The physical model for flow past a five-row deep in-line tube bank is shown in figure 20 . It is well established in the literature that, for relatively low Reynolds numbers and a closely packed bundle of cylinders, it is reasonable to assume that the wake behind each cylinder is symmetric. In fact, due to tight packing, no vortex shedding is likely to occur even up to moderate Reynolds numbers. It is further assumed that a line of symmetry exists as depicted in figure 20. The inflow boundary is located five cylinder diameters in front of the first row of cylinders. Similarly, in order to minimize the effect of outflow boundary condition on the fluid flow characteristics in the vicinity of the fifth cylinder, the computational domain has been extended up to 15 cylinder diameters in its downstream. In some sense, this problem is diametrically opposite to the disk and doughnut baffled heat exchanger, which was analysed in $\$ 4.2$. It should be pointed out that in $\S 4.2$, tubes that pass through the baffles and occupy the shell side were totally ignored in the flow path. On the contrary, in this section, baffles that pose resistance to flow path are completely ignored in the analysis. Thus, the objective is to obtain a local picture of resistance to fluid flow and heat transfer.

Boundary conditions - The following boundary conditions have been employed during the simulation.

(a) Cylinder surface - No slip $(U=V=0.0)$ and $\theta=1.0$ (isothermal tube surface);

(b) Axis of symmetry $-V=0.0$;

(c) Inlet - Uniform flow $(U=1.0, V=0.0)$ and $\theta=0.0$; and

(d) Exit $-P=0.0$.

\section{Initial conditions:}

$$
U=1.0 ; V=0.0 ; \theta=0.0 ; \text { at } \tau=0 .
$$

In this problem, the fluid flow patterns and heat transfer characteristics evolve with time before reaching the steady state. A detailed study on the temporal evolution of the flow

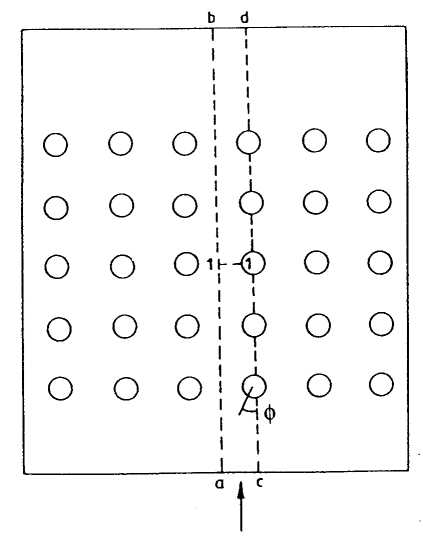

Figure 20. Configuration of the physical and computational (shown dotted) domains. 
(a)



(b)

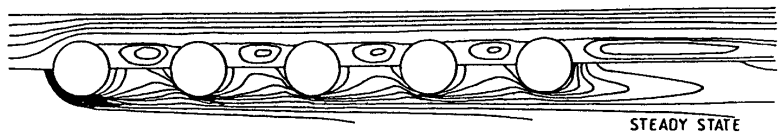

Figure 21. Streamlines and isotherms for (a) PDR $=1.5$ and (b) $\mathrm{PDR}=2.0$.

field is given by Krishne Gowda (1996). Streamline and isothermal patterns, after reaching the steady state, are depicted in figure 21, for two pitch-to-diameter ratios of 1.5 and 2.0, when $\operatorname{Re}=100$ and $\operatorname{Pr}=0.7$. Flow separates from the upstream cylinder and reattaches onto the downstream body. As can be observed, there are zones of recirculation in the gap region between cylinders. However, the region is much larger behind the last row of cylinders. Crowding of isotherms over the front half of the first row of cylinders is understandable, as the thermal boundary layer growth begins only from the first cylinder. For other rows, low velocity recirculating flow interacts with parts of the front half of the subsequent cylinders. Here, it should be emphasized that the heat flux between the third and fourth row of a bundle is equivalent to the heat flux between any two adjacent inner rows of an infinite bundle of cylinders.

Local skin friction coefficient is defined by,

$$
C_{f}=\left.\frac{2}{\operatorname{Re}} \frac{\partial U}{\partial Y}\right|_{Y=R},
$$

where, $\partial U / \partial Y$ is the velocity gradient evaluated on the cylinder surface. A plot of skin friction coefficient against angle $(\phi)$ is presented in figure 22 , for $\operatorname{Re}=100$ and PDR $=1.5$ and 2.0. The first cylinder offers higher viscous resistance than the rest of the tubes. This is intrinsic as the rest of the cylinders are submerged in the wake of their corresponding upstream cylinders. Comparison of skin friction coefficient is made with values given by
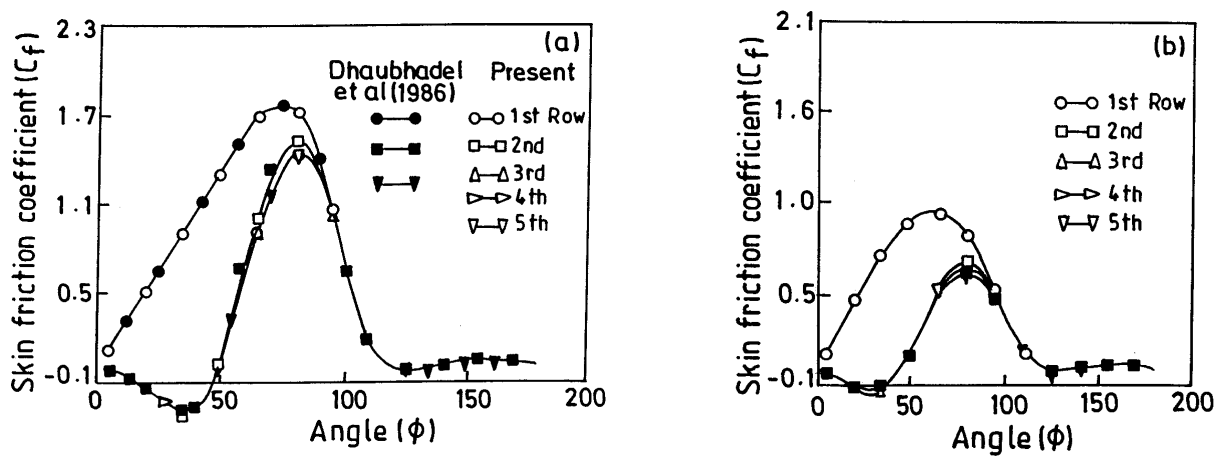

Figure 22. Variation of local skin friction coefficient (a) $\mathrm{PDR}=1.5$ and (b) $\mathrm{PDR}=2.0$. 

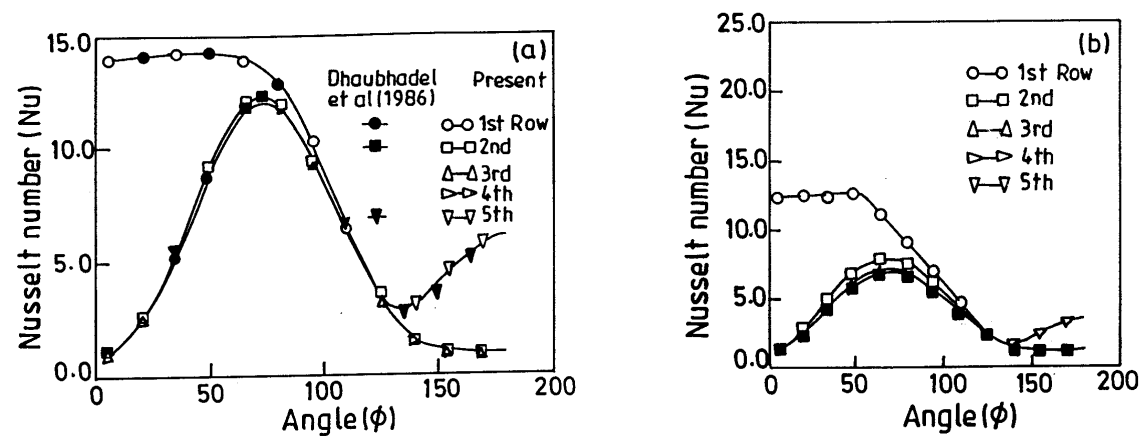

Figure 23. Variation of local Nusselt number for (a) PDR $=1.5$ and (b) PDR $=2.0$.

Dhaubhadel et al (1986) for PDR $=1.5$ and $\mathrm{Re}=100$. For $\mathrm{PDR}=2.0$, the plot is shown by figure $22 b$.

Nusselt number is one of the important design parameters of interest, which is given by the relation,

$$
\mathrm{Nu}=\left.\frac{\theta_{w}-\theta_{i n}}{\theta_{w}-\theta_{b}} \frac{\partial \theta}{\partial Y}\right|_{Y=R},
$$

where, $\theta_{w}$ is the wall temperature, $\theta_{b}$ is the bulk temperature at the minimum cross section, and $\theta_{\text {in }}$ refers to the inlet temperature. Figure 23 represents the distribution of local Nusselt number variation against angle $(\phi)$ for $\mathrm{PDR}=1.5$ and 2.0 at $\mathrm{Re}=100$. As can be observed, local Nusselt number distribution for the first row differs from those of the second and subsequent rows. This is due to the absence of wake shading influence on the first row of cylinders, unlike the rest. The thinner boundary layer over the first row of cylinders leads to a higher temperature gradient and, thus, higher heat transfer on its fore. The maximum $\mathrm{Nu}$ for the first row occurs at around $60^{\circ}$, which is in agreement with those obtained by Fuji et al (1984) and Dhaubhadel et al (1986). Over the first cylinder, the minimum $\mathrm{Nu}$ in the plot corresponds to the point of separation. For second and subsequent cylinders, there is a point of separation and reattachment as well. The zones of recirculation come into

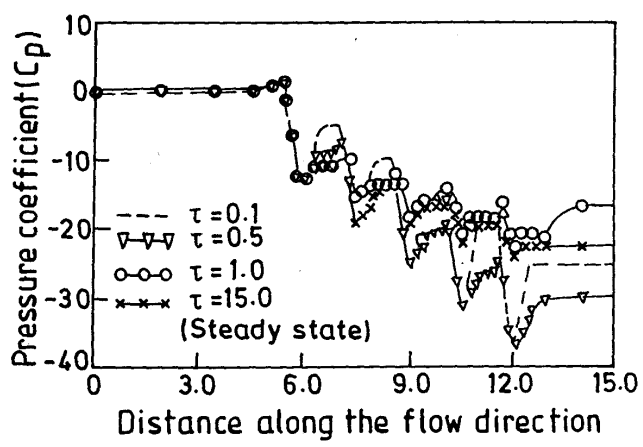

Figure 24. Pressure distribution around the cylinders for $\mathrm{PDR}=1.5 ; \mathrm{Re}=200$. 
contact on both sides, which justify increased resistance to heat flow and, hence, the smaller values of local $\mathrm{Nu}$.

Pressure distribution along CD of the computational domain at different time levels, during its evolution to steady state, is shown in figure 24. The pressure drop across the first row of cylinders is almost identical with the fore side of a single cylinder and remains unchanged up to steady state. From the second row onwards, pressure drop and recovery process take some time to reach steady state. Length of the recirculation zone behind the fifth row of cylinders has an influence on pressure drop and recovery process.

Conclusions: Laminar flow past a tube bank is numerically simulated in the low Reynolds number regime. Streamline patterns depict zones of recirculation between the cylinders. Local skin friction coefficient and Nusselt number variations are obtained, which are in good agreement with earlier investigations. Pressure distribution along the bundle crosssection is presented.

\section{Concluding remarks}

In the present study, laminar and turbulent flow simulations are carried out using a velocity correction algorithm. Four internal fluid flow problems with heat transfer are presented by solving full Navier-Stokes equations. Finite element-based Galerkin weighted residual formulation for spatial discretization and an Euler explicit scheme for temporal discretization are employed. The results of flow over a backward-facing step serve the purpose of extensive validation. Conjugate analysis is performed for flow through a disk and doughnut baffled heat exchanger. Simulation of this problem demonstrates the robustness of the code developed. Flow in a channel with circular, square and elliptic blockages is simulated. The influence of vortex dynamics on the Strouhal periodicity is examined. Simulation of laminar flow past a bundle of cylinders in a shell is carried out. Emphasis is given for the calculation of engineering parameters like Nusselt number, skin friction coefficient and pressure drop. Turbulent flow simulation for the latter class of flows, dominated by vortex dynamics, is possible only with a workable turbulence model. The versatility and robustness of the velocity correction algorithm is depicted in the successful simulation of a wide range of complex fluid flow features.

\section{List of symbols}

$\begin{array}{ll}C & \text { Courant number }(U \Delta \tau / \Delta X) ; \\ C_{f} & \text { skin friction coefficient; } \\ c_{p} & \text { specific heat of the fluid; } \\ C_{\mu}, C_{\varepsilon 1}, C_{\varepsilon 2} & \text { constants in } k-\varepsilon \text { turbulence model; } \\ D & \text { cylinder diameter; } \\ f_{\mu}, f_{1}, f_{2} & \text { functions in } k-\varepsilon \text { turbulence model; } \\ f_{v s} & \text { vortex shedding frequency; } \\ H & \text { channel height/ step height; } \\ k & \text { turbulent kinetic energy; } \\ k_{t} & \text { thermal conductivity of the fluid; } \\ l & \text { mixing length; }\end{array}$


$L_{\text {ref }} \quad$ reference length for non-dimensionalization;

$N_{i}, N_{j}, N_{k} \quad$ finite element shape functions;

$\mathrm{Nu}$

$P$

Nusselt number;

$\mathrm{Pe}$

static pressure;

$\mathrm{Pe}_{\mathrm{c}}$

Peclet number $\left(U L_{\mathrm{ref}} / \alpha\right)$;

$\operatorname{Pr}$

cell Peclet number $(U \Delta X / v)$;

$Q_{k}, Q_{\varepsilon}$

Prandtl number $\left(\mu c_{p} / k_{T}\right)$;

$\operatorname{Re}$

source terms for $k$ and $\varepsilon$ equations respectively;

St

Reynolds number $\left(U_{\text {ave }} L_{\text {ref }} / v\right)$;

$T_{\mathrm{ps}}$

Strouhal number $\left(f_{\mathrm{vs}} L_{\mathrm{ref}} / U_{\mathrm{ave}}\right)$;

$\overline{u_{i}^{\prime} u_{j}^{\prime}}$

time period of vortex shedding;

Reynolds stresses;

$U_{1}$

flow velocity along the streamwise direction;

$U_{2}, V$

$\tilde{U}_{i}$

flow velocity along the cross stream direction;

$\mathrm{U}_{\mathrm{av}}$

Fictitious or pseudo velocities;

$U_{y}$

$y$

$\alpha$ average velocity in a section;

monitored velocity of the signal traces;

normal wall distance;

thermal diffusivity ratio;

turbulent thermal diffusivity;

$\alpha_{T}$

$\alpha_{\text {rat }}$ thermal diffusivity ratio;

$\varepsilon$ dissipation of turbulent kinetic energy; inlet value of $\varepsilon$;

$\varepsilon_{\text {in }}$

$v$ momentum diffusivity; turbulent momentum diffusivity;

$\mathrm{v}_{T}$

$\phi$

$\rho$

$\sigma_{k}, \sigma_{\varepsilon}$

$\tau$

$\Delta \tau$

$\theta$ angle (measured from $X$-axis as the base);

density of the fluid;

model constants for diffusion of $k$ and $\varepsilon$;

dimensionless time; incremental time step;

temperature;

$\chi$

mixing length.

The authors thank the referees for their invaluable suggestions. One of the authors (BSVP) is supported by the National Science and Technology Board (NSTB) of the Republic of Singapore.

\section{References}

Autret A, Grandotto M, Dekeyser I 1987 Finite element computation of a turbulent flow over a twodimensional backward facing step. Int. J. Numer. Methods Fluids 7: 89-102

Benim A C, Zinser W 1985 Investigation into the finite element analysis of confined turbulent flows using a $k-\varepsilon$ model. Comput. Methods Appl. Mech. Engg. 51: 507-523

Blevins R D 1990 Flow induced vibrations (New York: Von Nostrand Reinhold)

Bradshaw P 1996 Understanding and prediction of turbulent flow - 1996. Int. J. Heat Fluid Flow 18: $45-54$ 
Braza M, Chassiang P, Ha Minh M 1986 Numerical study and physical analysis of pressure and velocity field in the near wake of a circular cylinder. J. Fluid Mech. 165: 79-130

Chilukuri R 1987 Incompressible laminar flow past a transversely vibrating cylinder. Trans. ASME J. Fluids Eng. 109: 166-171

Chorin A J 1968 Numerical solution of the Navier-Stokes equations. Math. Comput. 22: 745-762

Collins R J 1973 Band width reduction by automatic renumbering. Int. J. Numer. Methods Eng. 6: 345-356

Davis R W, Moore E F, Purtell L P 1984 A numerical and experimental study of confied flow around rectangular cylinders. Phys. Fluids 27: 46-59

Denham M K et al 1975 A directionally sensitive laser anemometer for velocity measurement in highly turbulent flows. J. Phys. 8: 681-683

Donea J, Ginliani S, Laval H, Quartapele 1982 Finite element solution of unsteady Navier-Stokes equations by fractional step method. Comput. Method Appl. Mech. Eng. 30: 53-73

Dhaubhadel M N, Reddy J N, Telionis D P 1986 Penalty finite element analysis of coupled fluid flow and heat transfer for in-line bundle of cylinders in cross flow. J. Non-linear Mech. 21: 361373

Durst F, Tropea C 1982 Flow over a two-dimensional backward facing step. IUTAM symposium on structures of complex turbulent shear flows (eds) R Dumas, L. Fulachier (New York: Springer), pp. 41-52

Eaton B E 1987 Analysis of laminar vortex shedding behind a circular cylinder by computer-aided flow visualization. J. Fluid Mech. 180: 117-145

Ferziger J H 1987 Simulation of incompressible turbulent flows. J. Comput. Phys. 69: 1-48.

Fletcher C A J 1984 Computational Galerkin methods (New York: Springer-Verlag)

Fujii M, Fujii T, Nagata T 1984 A numerical analysis of laminar flow and heat transfer of air to inline tube banks. Numer. Heat Trans. 7: 89-102

Gresho P M 1990 On the theory of semi-implicit projection methods for viscous incompressible flow and its implementation via a finite element method that also introduces a nearly consistent mass matrix Part 1: Theory, Int. J. Numer. Method Fluid 11: 587-620

Gresho P M, Chan S T, Lee R L, Upson C D 1984 A modified finite element method for solving the time-dependent incompressible Navier-Stokes equations Part 1: Theory. Int. J. Numer. Method Fluids 4: 557-598

Griffin O M, Votaw C W 1972 The vortex street in the wake of a vibrating cylinder. J. Fluid Mech. 51: $31-48$

Isomoto K, Honmd 1989 The effect of turbulent intensity on the reattachment process over a back ward facing step. Trans. ASME J. Fluids Eng. 111: 87-92

Jones W P, Launder B E 1972 The prediction of laminarization with a two-equation model of turbulence. Int. J. Heat Mass Trans. 15: 301-314

Karniadakis G M 1988 Numerical simulation of forced convection heat transfer from a cylinder in cross flow. Int. J. Heat Mass Transfer 31: 107-118

Kelkar K M, Patankar S V 1992 Numerical prediction of vortex shedding behind a square cylinder. Int. J. Numer. Method Fluids 14: 327-341

Kim J, Moin P 1985 Application of a fractional step method to incompressible Navier-Stokes equations. J. Comput. Phys. 59: 308-323

Kovacs A, Kawahara M 1991 A finite element scheme based on the velocity correction method for the solution of the time-dependent incompressible Navier-Stokes equations. Part 2: Applications. Int. J. Numer. Method Fluids 13: 403-423

Kovasznay L S G 1949 Hot wire investigation of wake behind cylinders at low Reynolds numbers. Proc. R. Soc. A198: 174-190

Krishne Gowda Y T 1996 finite element simulation of flow past tube banks with heat transfer. Ph D Thesis, Indian Institute of Technology, Chennai, India

Lam C K G, Bremhorst K 1981 A modified form of $k-\varepsilon$ model for predicting wall turbulence. Trans. ASME, J. Fluids Eng. 103: 456-460

Launder B E, Massey T H 1978 The numerical predictions of viscous flow and heat transfer in tube banks. Trans. ASME J. Heat Transfer 100: 565-571 
Launder B E, Sharma B L 1974 Application of the energy dissipation model of turbulence to the calculation of flow near a spinning disc. Lett. Heat Mass Trans 1: 131-145

Launder B E, Spalding B E 1972 Lectures in mathematical models of turbulence (London: Academic Press)

Leschziner M A 1993 Computational modelling of complex turbulent flow - expectations, reality and prospects. J. Wind Eng. Ind. Aeroyn. 46\&47: 37-51

Li H, Kottke V 1999 Analysis of local shell side heat and mass transfer in the shell and tube heat exchanger with disk and dough nut baffles. Int. J. Heat Mass Transfer 42: 3509-3521

Marvin J G, Huang G P 1998 Status and future directions for turbulence modelling. Sadhana, 23: 481-503

Mukhopadhyay A, Biswas G, Sundararajan T 1992 Numerical investigation of confined wakes behind a square cylinder in a channel. Int. J. Numer. Method Fluid 14: 1473-1484

Nallasamy M 1987 Turbulence models and their applications to the prediction of internal flows: A review. Comput. Fluids 15: 151-194

Natrajan R 1992 A numerical method for incompressible viscous flow simulation. J. Comput. Phys. 100: $384-395$

Ökajima A 1982 Strouhal number of rectangular cylinders. J. Fluid Mech. 123: 379-398

Ökajima A 1990 Numerical simulation of flow around rectangular cylinders. J. Wind Eng. Indian Aerodyn. 33: 171-180

Patnaik B S V 1994 Finite element analysis of flow past bluff bodies with heat transfer. MS thesis, Indian Institute of Technology, Chennai

Patnaik B S V 1998 Finite element analysis of flow past a circular cylinder and two cylinders in tandem: influence of vibration, buoyancy. Ph D thesis, Indian Institute of Technology, Chennai

Patnaik B S V, Seetharamu K N, Aswatha Narayana P A 1996 Simulation of laminar confined flow past a circular cylinder with an integral wake splitter plate involving heat transfer. Int. J. Numer. Method. Heat Fluid Flow 6: 65-81

Patnaik B S V, Aswatha Narayana P A, Seetharamu K N 1999a Numerical simulation of vortex shedding past a circular cylinder under the influence of buoyancy. Int. J. Heat Mass Transfer 42: 3495-3507

Patnaik B S V, Aswatha Narayana P A, Seetharamu K N 1999b Numerical simulation of laminar flow past a transversely vibrating circular cylinder. J. Sound Vib. 228: 459-475

Perkins H C, Leppert G 1964 Local heat transfer coefficients on a uniformly heated cylinder. Int. J. Heat Mass Transfer 7: 143-158

Perry A E, Chong M S, Lim T J 1982 The vortex shedding behind two-dimensional bluff bodies. $J$. Fluid Mech. 116: 77-90

Ramaswamy B, Jue T C, Akin JE 1992 Semi-implicit and explicit finite element schemes for coupled fluid/thermal problems. Int. J. Numer. Methods Eng. 34: 675-696

Ravikumaur S G 1988 Finite element analysis of convective heat transfer and heat exchangers. $\mathrm{Ph}$ D thesis, Indian Institute of Technology, Chennai

Ravisankar M S 1991 Finite element analysis of turbulent flows with heat transfer. MS thesis, Indian Institute of Technology, Chennai

Ren G, Utnes T 1993 A finite element solution of the time-dependent incompressible Navier-Stokes equations using a modified velocity correction method. Int. J. Numer. Methods Fluids 17: 349364

Roshko A 1993 Perspectives on bluff body aerodynamics. Int. J. Wind Eng. Ind. Aerodyn. 49: 79100

Rodi W 1982 Examples of turbulence models for incompressible flows. AIAA J. 20: 872-879

Rodi W 1984 Turbulence models and their applications in hydraulics - A state of the art review. 2nd edn. IAHR (Delft:)

Sa J Y, Chang K S 1991 Shedding patterns of near wake vortices behind a circular cylinder. Int. J. Numer. Methods Fluid 12: 463-474

Segarlind L J 1984 Applied finite element analysis (New York: Wiley)

Srinivas M 1994 Finite element analysis of internal flows with heat transfer $\mathrm{Ph} \mathrm{D}$ thesis, Indian Institute of Technology, Chennai 
Srinivas M, Ravisankar M S, Seetharamu K N, Aswatha Narayana P A 1994 Finite element analysis of internal flows with heat transfer. Sadhana 19: 785-816

Taylor C, Harper J J, Hughes T G, Morgan K 1981 An analysis of developing turbulent flow in a circular pipe by finite element method. Proc. Numer Methods Laminar Turbulence Flows (eds) Baker et al (Swansea: Pineridge)

Williamson C H K 1988 The existence of two stages in the transition to three dimensionality of a cylinder wake. Phys. Fluids 31: 3165-3168

Williamson C H K 1996 Vortex dynamics in the cylinder wake. Annu. Rev. Fluid Mech. 28: 477539

Zhang C, Sousa A C M 1990 Numerical simulation of turbulent shear flow in an isothermal heat exchanger model. Trans. ASME J. Fluids Eng. 112: 48-55

Zhu J, Sethian J 1992 Projection methods coupled to level set interface techniques. J. Comput. Phys. 102: $128-138$

Zienkiewicz O C, Philips D V 1971 An automatic mesh generation scheme for plane and curved surfaces by iso-parametric co-ordinates. Int. J. Numer. Methods Eng. 3: 519-528

Zukauskas A A 1987 Heat transfer from tubes in cross flow. Adv. Heat Transfer 18: 87-157 